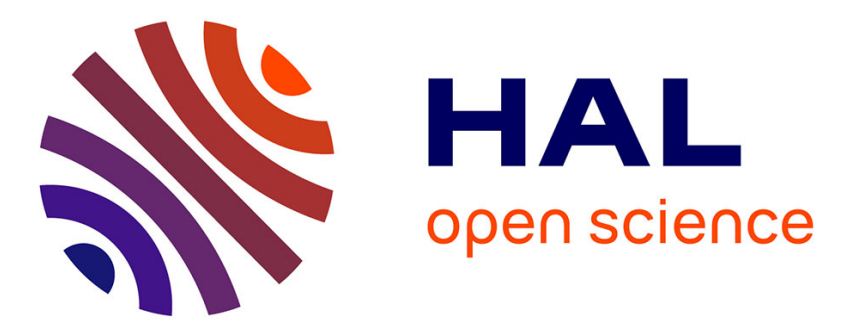

\title{
Macroscopic yield criteria for plastic anisotropic materials containing spheroidal voids
}

Vincent Monchiet, Djimedo Kondo, Eric Charkaluk, Cazacu Oana

\section{To cite this version:}

Vincent Monchiet, Djimedo Kondo, Eric Charkaluk, Cazacu Oana. Macroscopic yield criteria for plastic anisotropic materials containing spheroidal voids. International Journal of Plasticity, 2008, 24 (7), pp.1158-1189. 10.1016/j.ijplas.2007.08.008 . hal-00687823

\section{HAL Id: hal-00687823 https://hal.science/hal-00687823}

Submitted on 18 Apr 2012

HAL is a multi-disciplinary open access archive for the deposit and dissemination of scientific research documents, whether they are published or not. The documents may come from teaching and research institutions in France or abroad, or from public or private research centers.
L'archive ouverte pluridisciplinaire HAL, est destinée au dépôt et à la diffusion de documents scientifiques de niveau recherche, publiés ou non, émanant des établissements d'enseignement et de recherche français ou étrangers, des laboratoires publics ou privés. 


\title{
Macroscopic yield criteria for plastic anisotropic materials containing spheroidal voids
}

\author{
Vincent MONCHIET ${ }^{\mathrm{a}}$ Oana CAZACU ${ }^{\mathrm{b}}$ Eric CHARKALUK ${ }^{\mathrm{a}}$, \\ Djimedo KONDO a 1
}
${ }^{a}$ Laboratoire de Mécanique de Lille-UMR8107 CNRS, Université de Sciences et Technologies Lille,

Cité Scientifique, Bd. Paul Langevin, 59655 Villeneuve d'Ascq Cedex, France

${ }^{\mathrm{b}}$ Department of Mechanical and Aerospace Engineering, University of

Florida/REEF, 1350 N. Poquito Road Shalimar, FL 32579-1163, USA

\begin{abstract}
The combined effects of void shape and matrix anisotropy on the macroscopic response of ductile porous solids is investigated. The Gologanu-Leblond-Devaux's (GLD) analysis of an rigid-ideal plastic (von Mises) spheroidal volume containing a confocal spheroidal cavity loaded axisymmetrically is extended to the case when the matrix is anisotropic (obeying Hill's (1948) anisotropic yield criterion) and the representative volume element (RVE) is subjected to arbitrary deformation. To derive the overall anisotropic yield criterion, a limit analysis approach is used. Conditions of homogeneous boundary strain rate are imposed on every ellipsoidal confocal with the cavity. A two-field trial velocity satisfying these boundary conditions are considered. It is shown that for cylindrical and spherical void geometries, the proposed criterion reduces to existing anisotropic Gurson-like yield criteria. Furthermore, it is shown that for the case when the matrix is considered isotropic, the new results provide a rigorous generalization to the GLD model. Finally, the accuracy of the proposed approximate yield criterion for plastic anisotropic media containing non-spherical voids is assessed through comparison with numerical results.
\end{abstract}

Key words: , yield condition, anisotropic material, porous material, ideally plastic material, microstructures

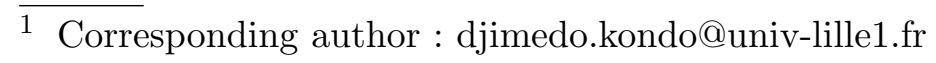




\section{Introduction}

During deformation, voids are nucleated in metals, mainly by decohesion at the hard particle-matrix interfaces. These voids grow in the matrix until coalescence, phenomenon that triggers ductile fracture. In the opposite direction, in the consolidation of metallic powders, plastic collapse of voids occurs when the material is compacted at certain rates and temperatures, to reduce porosity (Hom and McMeeking [20]). The evolution of a single void in an infinite isotropic rigid-perfect plastic matrix subjected to axisymmetric loading at the remote boundary was investigated by McClintock [28] for the case of cylindrical voids and Rice and Tracey [39] for spherical voids. However, voids in metallic alloys are often ellipsoidal. They are either prolate ellipsoids if they are nucleated around inclusions previously elongated during rolling (see for e.g. Benzerga et al. [4]) or oblate ellipsoids if they grow from cleavage cracks in the hard phase of a dual-phase structure (see for e.g. Son and Kim [42]). Lee and Mear (1992) extended the pioneering works of McClintock [28] and of Rice and Tracey [39] to ellipsoidal cavities embedded in an infinite viscous medium (obeying Norton's power law) and subjected to axisymmetric deformation. Key in their analysis is the solution of a kernel problem that provides the deformation fields (strain rates) in the matrix.

Based on the limit-analysis of a cavity embedded in a finite volume of an ideal rigid-plastic material obeying von Mises yield criterion and subjected to axisymmetric deformation, Gurson [18] has proposed analytical overall yield criteria for porous solids. Both spherical and cylindrical void geometries were considered. Despite the simplicity of the conceptual framework of limit-analysis methods, the yield surface obtained by Gurson constitutes an upper bound for porous media of the Hashin's "composite spheres assemblage" type (see [35]). An heuristic extension of the Gurson model has been proposed by Tveergard and Needleman [44] and used by many authors in several applications including cyclic plasticity (Perrin and Leblond [34], Bonora et al. [6], Pirondi et al. [36], etc.). Others extensions of the Gurson model concern the consideration of rate effects in the matrix (see for instance [24] or to effects of voids locking by inclusions [40], [41]). Very recently, studies on cylindrical void growth in rigid-ideally plastic single crystals (see [21], [22], [23]) have been reported.

Gologanu et al. [14], [15] and [17] generalized Gurson's analysis by considering a spheroidal volume containing a confocal spheroidal cavity. In Gãrãjeu [12] and Gãrãjeu et al. [13] a similar analysis was proposed for prolate cavities. In particular, the model of Gologanu et al.[14] has been applied by many authors [37], [38] or used for assessing toughness trends as function of void shape and spacing (e.g.[32],[33]) or for prediction of forming limits (e.g. Son and Kim [42]). It is to be noted that all the theories mentioned concern dilatational plasticity and ductile fracture in isotropic materials. However, as a result of forming processes, most metallic alloys display plastic anisotropy. Recent studies have been devoted to the experimental characterization of the anisotropy 
of fracture in different alloys (see for e.g. [4] for an overview of experimental evidence of anisotropic ductile fracture in steels) and to the formulation of anisotropic Gurson-like criteria for porous anisotropic sheet metals. For instance, Liao et al. [26] has proposed an analytic anisotropic Gurson-like yield criterion based on a limit analysis of a circular thin disk with a unique throughthickness void subjected to axisymmetric loading. The matrix was assumed to be rigid perfect plastic, incompressible, and obeying Hill's [19] quadratic anisotropic yield criterion. Furthermore, it was assumed that the anisotropy in the plane of the sheet is weak and can be described by a single anisotropy coefficient, R. Benzerga et al.[1-3] proposed an extension of Gurson model for the case when the matrix is fully orthotropic, its anisotropy being described by Hill's criterion with six independent coefficients. Assuming axisymmetric loading, closed-form approximate yield criteria were obtained for both spherical and cylindrical voids. Numerous numerical studies aimed at validating these anisotropic Gurson-like models were carried out (e.g. Chien et al. [9], Wang et al.[45], etc.). Generally, finite-element analyzes of a cube containing a spherical void, subjected to plane-stress conditions, performed for various initial porosities and different values of the anisotropy parameters are reported. More recently, these dilatational anisotropic plastic models have been used to predict forming limits for anisotropic sheets containing voids (see for example, Brunet et al. [7]). Note that all the anisotropic models cited can describe the response of porous media only for particular void shapes (spherical or cylindrical) and loading conditions. It should be expected that void shape has a significant influence on the behavior of porous anisotropic metals, yet there have been relatively few studies on the combined effects of void shape and texture (see for e.g the experimental study of Benzerga et al. [4]). The only attempts to model the overall behavior of anisotropic solids containing ellipsoidal voids are either heuristical or purely phenomenological (see [10], [5]). The main objective of this study is to provide an in-depth investigation of the role of void shape on the plastic deformation of anisotropic porous solids. More precisely, we seek to provide a closed-form approximate expression of a macroscopic yield criterion for anisotropic metals containing non spherical voids. To this end, a limit analysis of an ellipsoidal cavity embedded in a finite volume, namely a confocal ellipsoid is carried out. Rigid-plastic behavior obeying the 3-D Hill (1948) yield criterion is considered. The paper is organized as follows. Section 2 presents the statement of the problem and the homogenization adopted. Then, in section 3, we derive the overall yield criterion. As a first validation, in section 4 it is shown that for cylindrical and spherical void geometries, the proposed criterion reduces to existing anisotropic Gurson-like yield criteria. For the case of a von Mises matrix and axisymmetric loading, the proposed criterion reduces to that of Gologanu and co-workers [14], [15] and [17]. Furthermore, it provides a rigorous micromechanics-based generalization of these criteria to the case of arbitrary loading. Although limited to the case of isotropic matrix, the only micromechanics-based attempt to derive yield criteria for arbitrary loadings has been the one performed by Gologanu 
[16]. The Gologanu [16] formulation contains a very minor misprint which was pointed out and corrected in this work. To assess the accuracy of the proposed criterion, the general analytical yield loci were compared to 'exact' yield loci (corresponding to the two-field velocity) obtained by evaluating numerically the plastic dissipation. A very good agreement was observed. Incorporation of these results into a multiscale approach of high cycle fatigue can be found in Monchiet [29].

\section{- Notations}

The following notations are used for various tensors and their products.
a scalar
- simple contraction of two tensors
$\underline{a}$ vector
: double contraction of two tensors
a second order tensor
$\otimes$ tensorial product
A fourth order tensor
$\stackrel{s}{\otimes}$ symmetrized tensorial product
1 second order unit tensor
$\mathbb{I}=\mathbf{1} \bar{\otimes} \mathbf{1}$ symmetric fourth order unit tensor
$\mathbb{J} \quad=\frac{1}{3}(\mathbf{1} \otimes \mathbf{1})$
$\mathbb{K}=\mathbb{I}-\mathbb{J}$

$\overline{\boldsymbol{A}}$ deviator of a second order tensor $\boldsymbol{A} ; \quad A_{h}$ hydrostatic part of $\boldsymbol{A}$

\section{Statement of the problem}

An axisymmetric ellipsoidal cavity of semi-axes $a_{1}$ and $b_{1}$ embedded in a confocal ellipsoid of semi-axes $a_{2}$ and $b_{2}$ is considered. The choice of the geometry of the cell (Confocal spheroid) is such that it "conforms" to some extent to that of the void. Also such a choice allows to recover as a limit, the cases of coaxial cylindrical or spherical voids, respectively which are the only cells studied in the literature (see also Gologanu et al [14] for further discussion on the validity of this assumption). Figure 1 depicts the cell relative to the $\left(x_{1}, x_{2}, x_{3}\right)$ Cartesian coordinate system (of orthonormal basis $\left(e_{1}, e_{2}, e_{3}\right)$, with axis $x_{3}$ aligned with the axis of symmetry of the voids. The shape of the cavity is described by the aspect ratio $a_{1} / b_{1}$, with $a_{1}>b_{1}$ corresponding to a prolate cavity while $b_{1}>a_{1}$ corresponds to an oblate one. Let denote $c=\sqrt{\left|a_{1}^{2}-b_{1}^{2}\right|}=\sqrt{\left|a_{2}^{2}-b_{2}^{2}\right|}$ the focal distance and $e_{1}$ the void eccentricity defined by:

$$
e_{1}=\frac{c}{a_{1}} \quad \text { (prolate); } \quad e_{1}=\frac{c}{b_{1}} \quad \text { (oblate) }
$$

We will use both cylindrical coordinates $\rho, \theta, \mathrm{z}$ and $\left(e_{\rho}, e_{\theta}, e_{z}\right)$ the associated 


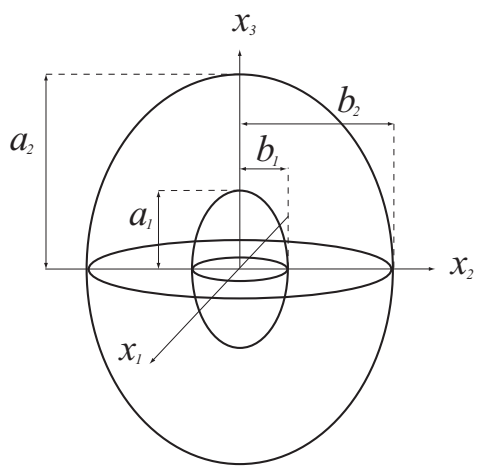

$a$

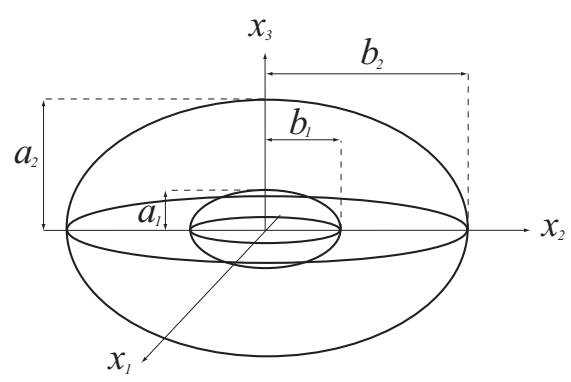

$b$

Fig. 1. The cell considered (a) prolate and (b) oblate spheroidal void embedded in a confocal spheroid relative to a Cartesian coordinate system $\left(x_{1}, x_{2}, x_{3}\right)$.

orthonormal basis and the classical elliptical coordinates $\lambda, \varphi, \theta$ (associated orthogonal basis $\left(e_{\lambda}, e_{\varphi}, e_{\theta}\right)$ shown in Figure 2) defined by (see [31]):

$$
\left\{\begin{array} { l } 
{ x _ { 1 } = b \operatorname { s i n } ( \varphi ) \operatorname { c o s } ( \theta ) } \\
{ x _ { 2 } = b \operatorname { s i n } ( \varphi ) \operatorname { s i n } ( \theta ) } \\
{ x _ { 3 } = a \operatorname { c o s } ( \varphi ) }
\end{array} \quad \left\{\begin{array}{l}
\rho=b \sin (\varphi) \\
\theta=\theta \\
z=x_{3}=a \cos (\varphi)
\end{array}\right.\right.
$$

and

$$
\left\{\begin{array}{l}
\underline{e}_{\lambda}=\frac{1}{L_{\lambda}}\left\{a \sin (\varphi) \underline{e}_{\rho}+b \cos (\varphi) \underline{e}_{3}\right\} \\
\underline{e}_{\varphi}=\frac{1}{L_{\lambda}}\left\{b \cos (\varphi) \underline{e}_{\rho}-a \sin (\varphi) \underline{e}_{3}\right\} \\
\underline{e}_{\theta}=\underline{e}_{\theta}
\end{array}\right.
$$

with $L_{\lambda}=\sqrt{a^{2} \sin ^{2}(\varphi)+b^{2} \cos ^{2}(\varphi)}, \lambda \in\left[0,+\infty\left[, \varphi \in[0, \pi]\right.\right.$ and $\underline{e}_{\rho}=\cos (\theta) \underline{e}_{1}+$ $\sin (\theta) \underline{e}_{2}, \theta \in[0,2 \pi]$.

In the above equations: $a=c \cosh (\lambda)$ and $b=c \sinh (\lambda)$ for a prolate void, while for the case of an oblate void: $a=c \sinh (\lambda)$ and $b=c \cosh (\lambda)$. The iso- $\lambda$ surfaces are confocal spheroids with foci on the circle $\rho=c, z=0$.

The porosity $f$ and the void shape parameter $S$ are given, respectively, by:

$$
f=\frac{a_{1} b_{1}^{2}}{a_{2} b_{2}^{2}}=\frac{\omega}{\Omega} ; \quad S=\ln \left(\frac{a_{1}}{b_{1}}\right)
$$

where $|\omega|$ represents the volume occupied by the spheroidal void and $|\Omega|$ denotes the volume of the studied cell. Since the exterior and interior spheroids 


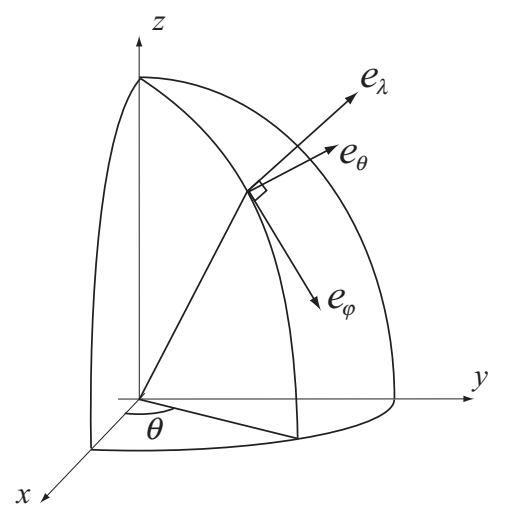

Fig. 2. Spheroidal coordinates of a prolate void

are confocal, the porosity $f$ can be expressed in terms of the eccentricities $e_{1}$ and $e_{2}$ as:

$$
f= \begin{cases}\frac{e_{2}^{3}}{e_{1}^{3}} \frac{1-e_{1}^{2}}{1-e_{2}^{2}} ; & \text { (prolate void) } \\ \frac{e_{2}^{3}}{e_{1}^{3}} \frac{\sqrt{1-e_{1}^{2}}}{\sqrt{1-e_{2}^{2}}} ; & \text { (oblate void) }\end{cases}
$$

The representative cell studied is assumed to be subjected to arbitrary loading and homogeneous boundary strain rate conditions i.e.

$$
\underline{v}\left(\lambda=\lambda_{2}\right)=\boldsymbol{D} \cdot \underline{x}
$$

where $\boldsymbol{D}$ denotes the macroscopic strain rate tensor, $\underline{v}$ is the velocity field, and $\mathrm{x}$ the current position in the matrix of the unit cell. A limit analysis approach will be used to obtain the overall yield criterion of the porous solid. Let denote by $\Pi(\boldsymbol{D})=\boldsymbol{\Sigma}: \boldsymbol{D}$, the macroscopic plastic dissipation. For all macroscopic stresses $\boldsymbol{\Sigma}$ and macroscopic strain rates $\boldsymbol{D}$ (see for example, [43]):

$$
\Pi(\boldsymbol{D})=\operatorname{Inf}<\pi(\boldsymbol{d}(\underline{x}))>_{\Omega}
$$

In the above equation, $\pi(\boldsymbol{d})$ is the local plastic dissipation defined as: $\pi(\boldsymbol{d})=$ $\boldsymbol{\sigma}: \boldsymbol{d}$ with $\boldsymbol{\sigma}$ and $\boldsymbol{d}$, the local stress and strain rate fields, respectively; $<>_{\Omega}$ stands for an average value taken over the domain of the cell, $\Omega$, and the infimum (Inf) is taken over all incompressible velocity fields kinematically admissible (i.e. satisfying the condition (6)). It follows that $\Pi(\boldsymbol{D})$ is obtained by integrating $\pi(\boldsymbol{d})$ over $\Omega$. The macroscopic stress at yielding corresponds to 


$$
\boldsymbol{\Sigma}=\frac{\partial \Pi}{\partial \boldsymbol{D}}
$$

\section{Approximate yield criterion for anisotropic media containing spheroidal cavities}

In this section, we derive the macroscopic yield criterion for an orthotropic material containing spheroidal cavities. A crucial step consists in finding a velocity field compatible with uniform strain rate boundary conditions. We begin by presenting in section 3.1 the velocity field that describes the expansion of the spherical cavity. Evaluation of the macroscopic plastic dissipation is presented in Section 3.2.

\subsection{Trial velocity field for a spheroidal cavity}

As already mentioned, a crucial step in the analysis is finding a velocity field that describes the expansion of the cavity. Such field must comply with both the incompressibility condition of the solid matrix, i.e. $\operatorname{div}(\underline{v})=0$, and with the uniform strain rate condition on the exterior boundary of the cell. Following Gologanu et al. [14], [15], we seek a trial velocity field, $\underline{v}$, of the form:

$$
\underline{v}=\boldsymbol{A} \cdot \underline{x}+B \underline{v}^{E}
$$

where $\boldsymbol{A}$ is a constant (uniform) second-order tensor and $B$ is a constant. A velocity field $\underline{v}^{E}$ that is incompressible, was proposed by Lee and Mear [25]. Imposing uniform strain rate boundary conditions, i.e. $\underline{v}=\boldsymbol{D} . \underline{x}$ on the exterior boundary of the spheroidal cell $\lambda=\lambda_{2}$, it follows that the velocity field in the matrix is given by (see [14], [15]):

$$
\begin{aligned}
& \underline{v}=\boldsymbol{A} \cdot \underline{x}+\frac{c^{3} B}{b L_{\lambda}}\left\{1+(1-3 \alpha) \frac{3 \cos ^{2}(\varphi)-1}{2}\right\} \underline{e}_{\lambda} \\
& -\frac{3 c^{3} B}{4 a b^{2} L_{\lambda}}\left(a^{2}-b^{2}\right)(1-\alpha-\beta) \sin (2 \varphi) \underline{e}_{\varphi}
\end{aligned}
$$

where

$$
\begin{aligned}
& \boldsymbol{A}=\boldsymbol{D}-D_{h} \boldsymbol{X} ; \quad B_{00}=6 B_{22}=B=\frac{a_{2} b_{2}^{2}}{c^{3}} D_{h} \\
& \text { with }: \quad \boldsymbol{X}=\frac{3}{2}\left(1-\alpha_{2}\right)\left(\underline{e}_{1} \otimes \underline{e}_{1}+\underline{e}_{2} \otimes \underline{e}_{2}\right)+3 \alpha_{2} \underline{e}_{3} \otimes \underline{e}_{3}
\end{aligned}
$$


and $D_{h}=\frac{1}{3} \operatorname{tr}(\boldsymbol{D})$. Tensor $\boldsymbol{X}$ depends only on $\alpha_{2}=\alpha\left(e_{2}\right)$, i.e. on the exterior boundary of the unit cell.

The scalar functions $\alpha$ and $\beta$ depending on $e$ are defined by:

$$
\begin{aligned}
& \alpha(e)= \begin{cases}\frac{a b^{2}}{c^{3}} \operatorname{arctanh}\left\{\frac{c}{a}\right\}-\frac{b^{2}}{c^{2}} & \text { (prolate void) } \\
-\frac{a b^{2}}{c^{3}} \arctan \left\{\frac{c}{a}\right\}+\frac{b^{2}}{c^{2}} & \text { (oblate void) }\end{cases} \\
& \beta(e)= \begin{cases}(1-3 \alpha) \frac{a^{2}}{c^{2}} & \text { (prolate void) } \\
-(1-3 \alpha) \frac{a^{2}}{c^{2}} & \text { (oblate void) }\end{cases}
\end{aligned}
$$

The introduction of the functions $\alpha(e)$ and $\beta(e)$ allow to use the same methodology for both oblate and prolate cavities. The variation of $\alpha$ and $\beta$ with the eccentricity $e$ is shown on Figure 3. In Table 1 are indicated the values taken by $\alpha$ and $\beta$ in some particular cases of special interest for which the eccentricity $e$ is constant i.e. that of cylindrical voids $\left(e_{1} \rightarrow 1\right.$ and $e_{2} \rightarrow 1$ in the case of prolate); sandwich ( $e_{1} \rightarrow 1$ and $e_{2} \rightarrow 1$ in the case of oblate). The spherical cavity $\left(e_{1} \rightarrow 0\right.$ and $\left.e_{2} \rightarrow 0\right)$ can be studied as a prolate or oblate.
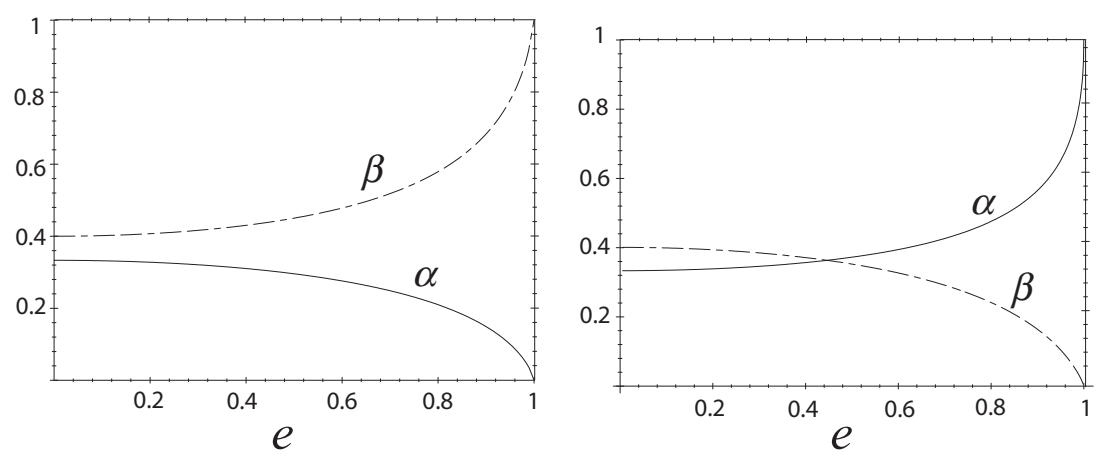

Fig. 3. Representation of the variation of $\alpha$ and $\beta$ with the eccentricity $e$ for: (a) a prolate void and (b) an oblate void.

\begin{tabular}{|c|c|c|c|}
\hline & cylindrical & spherical & sandwich \\
\hline$\alpha$ & 0 & $1 / 3$ & 1 \\
\hline$\beta$ & 1 & $2 / 5$ & 0 \\
\hline
\end{tabular}

Table 1: The values of the functions $\alpha$ and $\beta$ for some particular void shapes.

From (10) it is readily seen that the strain rate field in the matrix is the sum of an homogenous deviatoric field $\boldsymbol{A}$ and a non-homogeneous field $\boldsymbol{d}^{E}$ : 


$$
\boldsymbol{d}=\boldsymbol{A}+B \boldsymbol{d}^{E}
$$

with $\boldsymbol{d}^{E}$ given by:

$$
\begin{aligned}
& \boldsymbol{d}^{E}=\frac{3 c^{3}}{2 a b^{2}}(1-\alpha)\left(\mathbf{1}-3 \underline{e}_{\lambda} \otimes \underline{e}_{\lambda}\right)+\frac{3 c^{5} \beta}{2 a b^{2} L_{\lambda}^{2}} \sin ^{2}(\varphi)\left(\underline{e}_{\lambda} \otimes \underline{e}_{\lambda}-\underline{e}_{\varphi} \otimes \underline{e}_{\varphi}\right) \\
& +\frac{3 c^{5} \beta}{2 a^{2} b L_{\lambda}^{2}} \sin (2 \varphi) \underline{e}_{\lambda} \stackrel{s}{\otimes} \underline{e}_{\varphi}
\end{aligned}
$$

It can be easily shown that for the particular cases of spherical and cylindrical cavities, this strain rate field reduces to the classical one used by Gurson [18]. It can be argued that in the case when the matrix material is anisotropic, the use of the trial velocity defined by (10) with $\boldsymbol{A}$ given by (11) is questionable. Indeed, due to the anisotropy of the matrix, the exact solution to the problem of cavity expansion cannot be radial. The derivation of a non-radial trial velocity field remains an unresolved problem. Thus, in the case of anisotropic matrix a radial field is used (e.g. by [2] for spherical voids and by [26] for cylindrical ones). As already mentioned, neither an exact nor an approximate solution exist for the general problem of a hollow rigid-plastic orthotropic spheroid subjected to arbitrary loading ${ }^{2}$. Nevertheless, by adopting this trial velocity field, the resulting approximate yield surface is external to the exact yield surface.

3.2 Evaluation of the macroscopic plastic dissipation for an orthotropic matrix and a spheroidal cavity

We assume that the matrix material is rigid-plastic and satisfies Hill [19] yield criterion. Let denote by $\boldsymbol{\sigma}$ the stress field in the matrix. With respect to a Cartesian coordinate system associated with the axes of orthotropy, Hill [19] yield criterion is given by:

$$
F(\boldsymbol{\sigma})=\frac{3}{2} \boldsymbol{\sigma}: \mathbb{M}: \boldsymbol{\sigma}-\sigma_{0}^{2} \leq 0
$$

where $\sigma_{0}$ is a material constant and $\mathbb{M}$ is an orthotropic fourth-order symmetric tensor satisfying both major and minor symmetries (i.e. $M_{i j k l}=M_{j i k l}=$

$\overline{2}$ In a very particular cases of spherical voids in a transversely isotropic matrix or of a cylindrical void in an orthotropic matrix, a field inspired from the solution of Eshelby equivalent inclusion problem can be choosen as a first attempt to generalize the results of this study. Such an approach was very recently investigated by Monchiet et al. [30] in the case of an isotropic matrix. 
$M_{k l j i}$ for $\left.i, j, k, l=1,2,3\right)$. To ensure plastic incompressibility, $M_{i i k l}=0$ for $k, l=1,2,3$ (summation after $i$ ). Thus, in the Cartesian coordinate system $\left(\underline{w}_{1}, \underline{w}_{2}, \underline{w}_{3}\right)$ associated with the axes of orthotropy, $\mathbb{M}$ has only six non-zero components, $F, G, H, L, M$ and $N$ :

$$
\mathbb{M}^{\text {ortho }}=\left(\begin{array}{cccccc}
H+G & -H & -G & 0 & 0 & 0 \\
-H & F+H & -F & 0 & 0 & 0 \\
-G & -F & F+G & 0 & 0 & 0 \\
0 & 0 & 0 & N & 0 & 0 \\
0 & 0 & 0 & 0 & M & 0 \\
0 & 0 & 0 & 0 & 0 & L
\end{array}\right)
$$

In the case when the cavity is arbitrarily oriented with respect to the axes of orthotropy, the tensor $\mathbb{M}$ is represented in the spheroidal coordinate system $\left(\underline{e}_{1}, \underline{e}_{2}, \underline{e}_{3}\right)$ by a matrix of 21 non-zero components:

$$
M_{i j k l}=R_{i m} R_{j n} R_{k p} R_{l q} M_{m n p q}^{\text {ortho }}
$$

where $\boldsymbol{R}$ is a second-order orthogonal tensor whose components in the $\left(\underline{w}_{1}, \underline{w}_{2}, \underline{w}_{3}\right)$ frame are (see Figure 4 for the definition of the angles $\phi$ and $\psi$ ):

$$
R=\left(\begin{array}{ccc}
\cos (\phi) \cos (\psi) & -\sin (\phi) & \cos (\phi) \sin (\psi) \\
\sin (\phi) \cos (\psi) & \cos (\phi) & \sin (\phi) \sin (\psi) \\
-\sin (\psi) & 0 & \cos (\psi)
\end{array}\right)
$$

When the matrix is isotropic i.e. $F=G=H=1 / 3$ and $L=M=N=1$ (15) reduces to von Mises yield criterion. Assuming an associated flow rule and denoting the plastic multiplier by $\dot{\Lambda}$, the strain rate in the matrix is given by:

$$
\boldsymbol{d}=\dot{\Lambda} \frac{\partial F}{\partial \boldsymbol{\sigma}}=3 \dot{\Lambda} \mathbb{M}: \boldsymbol{\sigma} \quad \text { when } \quad F(\boldsymbol{\sigma})=0
$$

Let $\mathbb{K}=\mathbb{I}-\mathbb{J}$, where $\mathbb{I}$ is the fourth-order symmetric identity tensor and $\mathbb{J}=\frac{1}{3} \mathbf{1} \otimes \mathbf{1}$. Let $\mathbb{H}$ be a fourth-order symmetric tensor satisfying both major and minor symmetries and ensure plastic incompressibility, $M_{i i k l}=0$ for $k, l$ 


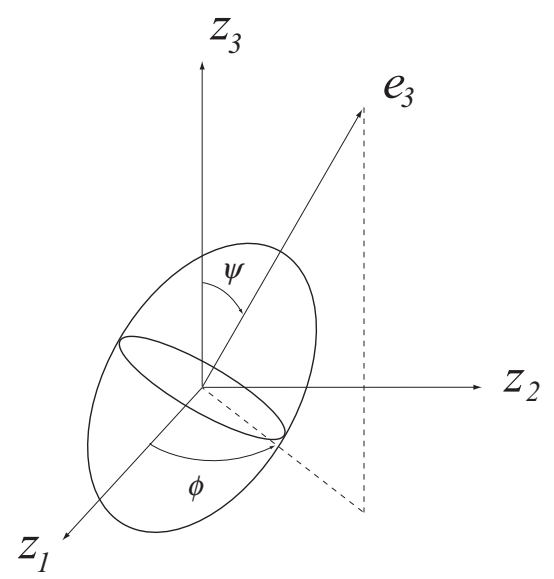

Fig. 4. Definition of the angles $\phi$ and $\psi$ characterizing the orientation of the spheroidal cavity with respect to the Cartesian coordinate system associated with the axes of orthotropy of the matrix

$=1,2,3$, such that: $\mathbb{H}: \mathbb{M}=\mathbb{M}: \mathbb{H}=\mathbb{K}$. Then, from the flow rule (19), it follows that:

$$
\overline{\boldsymbol{\sigma}}=\frac{1}{3 \dot{\Lambda}} \mathbb{H}: \mathbf{d}
$$

where $\overline{\boldsymbol{\sigma}}$ is the stress deviator. Enforcing the yield condition, we obtain ${ }^{3}$ :

$$
\dot{\Lambda}=\frac{d_{e q}}{2 \sigma_{0}} ; \quad \text { with }: \quad d_{e q}=\left[\frac{2}{3} \boldsymbol{d}: \mathbb{H}: \boldsymbol{d}\right]^{1 / 2}
$$

On the other hand, the plastic dissipation on the unit cell is given by:

$$
\pi(\boldsymbol{d})= \begin{cases}\sigma_{0} d_{e q} \quad & \text { (in the matrix) } \\ 0 \quad & \text { (in the voids) }\end{cases}
$$

In the coordinate system associated with the matrix orthotropy, $\mathbb{H}$ has six nonzero components which are expressible in terms of the anisotropy coefficients $F, G, H, L, M, N$ :

3 Note that in the case of an isotropic matrix i.e. $\mathbb{M}=\mathbb{H}=\mathbb{K}, d_{\text {eq }}=\left[\frac{2}{3} \boldsymbol{d}: \boldsymbol{d}\right]^{1 / 2}$ i.e. the effective plastic strain associated to the von Mises yield criterion. 


$$
\begin{array}{rlrl}
H_{11}^{\text {ortho }} & =\frac{1}{9} \frac{H+4 F+G}{F G+F H+G H} ; & H_{22}^{\text {ortho }} & =\frac{1}{9} \frac{H+F+4 G}{F G+F H+G H} \\
H_{33}^{\text {ortho }} & =\frac{1}{9} \frac{4 H+F+G}{F G+F H+G H} ; & H_{12}^{\text {ortho }} & =\frac{1}{9} \frac{H-2 F-2 G}{F G+F H+G H} \\
H_{13}^{\text {ortho }} & =\frac{1}{9} \frac{-2 H-2 F+G}{F G+F H+G H} ; & H_{23}^{\text {ortho }} & =\frac{1}{9} \frac{-2 H+F-2 G}{F G+F H+G H} \\
H_{44}^{\text {ortho }} & =\frac{1}{N} ; \quad H_{55}=\frac{1}{M} ; \quad H_{66}^{0}=\frac{1}{L}
\end{array}
$$

Using for the strain rate in the anisotropic plastic matrix, the trial field (13), $d_{e q}$ reads:

$$
d_{e q}^{2}=A_{e q}^{2}+\frac{4}{3} B \boldsymbol{A}: \mathbb{H}: \boldsymbol{d}^{E}+d_{e q}^{E^{2}} B^{2},
$$

where

$$
A_{e q}^{2}=\frac{2}{3} \boldsymbol{A}: \mathbb{H}: \boldsymbol{A}, \text { and } d_{e q}^{E}=\frac{2}{3} \boldsymbol{d}^{E}: \mathbb{H}: \boldsymbol{d}^{E}
$$

Hence, the macroscopic plastic dissipation is given by:

$$
\begin{aligned}
\Pi(\boldsymbol{D}) & =\frac{1}{|\Omega|} \int_{\Omega-\omega} \sigma_{0} d_{e q} d V \\
& =\frac{3 \sigma_{0}}{4 \pi a_{2} b_{2}^{2}} \int_{\lambda=\lambda_{1}}^{\lambda=\lambda_{2}} \int_{\varphi=0}^{\varphi=\pi} \int_{\theta=0}^{\theta=2 \pi} d_{e q} b L_{\lambda}^{2} \sin \varphi d \lambda d \varphi d \theta
\end{aligned}
$$

Let us introduce the following notation:

$$
<G(\varphi, \theta)>_{\mathcal{E}(\lambda)}=\frac{3}{4 \pi\left(2 a^{2}+b^{2}\right)} \int_{\varphi=0}^{\varphi=\pi} \int_{\theta=0}^{\theta=2 \pi} G(\varphi, \theta) L_{\lambda}^{2} \sin (\varphi) d \varphi d \theta
$$

which represents an average value of $G$ taken over the surface of a confocal spheroid $\mathcal{E}(\lambda)$.

Making use of the change of variable: $x=\frac{c^{3}}{a b^{2}}$, the macroscopic plastic dissipation becomes:

$$
\Pi(\boldsymbol{D})=-\sigma_{0} x_{2} \int_{x=x_{1}}^{x=x_{2}}<d_{e q}>_{\mathcal{E}(\lambda)} \frac{d x}{x^{2}}
$$


3.3 Approximate closed form expression of the macroscopic plastic dissipation $\Pi(\boldsymbol{D})$

The difficulties associated with obtaining a closed-form expression of the macroscopic plastic dissipation in the case of an isotropic matrix have been extensively discussed in the literature (see for example, Gurson [18], Gologanu et al., $[14,15,17]$, etc). In the case of spheroidal cavities embedded in a von Mises plastic matrix, a procedure has been already proposed by Gologanu et al. [14]. Consideration of the plastic anisotropy of the matrix makes this procedure unapplicable. In this work, a new procedure is proposed. In the isotropic case, it reduces to the one introduced by Gologanu et al.. As in the isotropic case, the proposed procedure involves certain approximations which, unfortunately, do not preserve the "upper bound" character of the results (except for the case of a spherical or a cylindrical void). The first approximation that we make is:

$\mathcal{A} 1: d_{e q}$ is replaced by $\left[<d_{e q}^{2}>_{\mathcal{E}(\lambda)}\right]^{1 / 2}$, its mean value along each spheroid confocal to the cavity.

Assuming $\mathcal{A} 1$, the expression $(28)$ of $\Pi(\boldsymbol{D})$ becomes:

$$
\Pi(\boldsymbol{D})=-\sigma_{0} x_{2} \int_{x_{1}}^{x_{2}}\left[<d_{e q}^{2}>_{\mathcal{E}(\lambda)}\right]^{1 / 2} \frac{d x}{x^{2}}
$$

Recalling that $\boldsymbol{A}$ is uniform and that the anisotropy tensor $\mathbb{H}$ is constant, it follows that:

$$
<d_{e q}^{2}>_{\mathcal{E}(\lambda)}=A_{e q}^{2}+\frac{4}{3} \boldsymbol{A}: \mathbb{H}:<\boldsymbol{d}^{E}>_{\mathcal{E}(\lambda)} B+<d_{e q}^{E^{2}}>_{\mathcal{E}(\lambda)} B^{2}
$$

The computation of $\Pi(\boldsymbol{D})$ by using (29) requires some additionnal approximations. The general procedure allowing to obtain a closed form expression of $\Pi(\boldsymbol{D})$ as well as the required approximations are presented in appendix 6.1. Using the approximations $\mathcal{A} 1$ to $\mathcal{A} 4$ (described in Appendix 6.1), it is readily seen that $<d_{e q}^{2}>_{\mathcal{E}(\lambda)}$ has the form given by (95) and thus, the closed-form approximation of the macroscopic plastic dissipation is:

$$
\begin{gathered}
\Pi(\boldsymbol{D})=-\sigma_{0} x_{2} \int_{u_{1}}^{u_{2}}\left\{\widetilde{A}^{2}+\tilde{B}^{2} u^{2}\right\}^{1 / 2} \frac{d u}{u^{2}} \\
=-\sigma_{0} x_{2}\left[\widetilde{B} \operatorname{arcsinh}\left\{\frac{u \widetilde{B}}{\widetilde{A}}\right\}-\frac{\sqrt{\widetilde{A}^{2}+u^{2} \widetilde{B}^{2}}}{u}\right]_{u_{1}}^{u_{2}}
\end{gathered}
$$


where:

$$
\widetilde{A}=\left[A_{e q}^{2}-\frac{w^{2} u_{1} u_{2}}{p^{2}}(\boldsymbol{A}: \mathbb{H}: \boldsymbol{Q})^{2}\right]^{1 / 2} ; \quad \widetilde{B}=p B-\frac{w}{p} \boldsymbol{A}: \mathbb{H}: \boldsymbol{Q}
$$

where $\boldsymbol{Q}$ is the deviatoric second-order tensor defined by ( 81$)$.

\subsection{Analytical expression of the approximate macroscopic yield criterion}

The macroscopic stresses associated to the yield locus are derived from the plastic dissipation $\Pi(\boldsymbol{D})$ (see $(31)$ ). We first express the partial derivatives of $\Pi(\boldsymbol{D})$ with respect to $\widetilde{A}$ et $\widetilde{B}$. Using (31), we obtain:

$$
\begin{aligned}
& \frac{\partial \Pi}{\partial \widetilde{B}}=-\sigma_{0} x_{2}[\operatorname{arcsinh}(u \xi)]_{u_{1}}^{u_{2}} \\
& \frac{\partial \Pi}{\partial \widetilde{A}}=-\sigma_{0} x_{2}\left[\frac{\sqrt{1+u^{2} \xi^{2}}}{u}\right]_{u_{1}}^{u_{2}}
\end{aligned}
$$

from which is derived the following expression of the criterion:

$$
\frac{1}{\sigma_{0}^{2}}\left(\frac{\partial \Pi}{\partial \widetilde{A}}\right)^{2}+2 \frac{x_{2}^{2}}{u_{1} u_{2}} \cosh \left(\frac{1}{x_{2} \sigma_{0}} \frac{\partial \Pi}{\partial \widetilde{B}}\right)-\frac{x_{2}^{2}}{u_{2}^{2}}-\frac{x_{2}^{2}}{u_{1}^{2}}=0
$$

where $\xi=\widetilde{B} / \widetilde{A}$. Let us recall that for a prolate void $u_{2}=x_{2}$ and $u_{1}=x_{1}=$ $x_{2} / f$ while for an oblate one:

$$
u_{2}=y_{2}=\frac{g}{\rho(1+g)} \quad \text { and } \quad u_{1}=y_{1}=\frac{g}{\rho(g+f)}
$$

with: $g=\rho x_{2}$ (see Appendix 6.2 for the definition of coefficient $\rho$ ) where $x=c^{3} /\left(a b^{2}\right)$. To further specify the yield criterion, we need to express $\frac{\partial \Pi}{\partial \widetilde{A}}$ and $\frac{\partial \Pi}{\partial \widetilde{B}}$ in terms of the macroscopic stress tensor.

3.4.1 Determination of $\frac{\partial \Pi}{\partial \widetilde{A}}$ and $\frac{\partial \Pi}{\partial \widetilde{B}}$

Since, (see (11)): 


$$
\begin{aligned}
& \boldsymbol{D}=\boldsymbol{A}+D_{h} \boldsymbol{X} ; \quad B=\frac{a_{2} b_{2}^{2}}{c^{3}} D_{h} \\
& \text { with : } \boldsymbol{X}=\frac{3}{2}\left(1-\alpha_{2}\right)\left(\underline{e}_{1} \otimes \underline{e}_{1}+\underline{e}_{2} \otimes \underline{e}_{2}\right)+3 \alpha_{2} \underline{e}_{3} \otimes \underline{e}_{3}
\end{aligned}
$$

it follows that:

$$
\begin{aligned}
& 3 \Sigma_{h}=\frac{\partial \Pi}{\partial D_{h}}=\frac{\partial \Pi}{\partial \boldsymbol{A}}:(\mathbf{1}-\boldsymbol{X})+\frac{\partial \Pi}{\partial B} \frac{a_{2} b_{2}^{2}}{c^{3}} \\
& \overline{\boldsymbol{\Sigma}}=\frac{\partial \Pi}{\partial \overline{\boldsymbol{D}}}=\frac{\partial \Pi}{\partial \boldsymbol{A}}: \frac{\partial \boldsymbol{A}}{\partial \overline{\boldsymbol{D}}}+\frac{\partial \Pi}{\partial B} \frac{\partial B}{\partial \overline{\boldsymbol{D}}}=\frac{\partial \Pi}{\partial \boldsymbol{A}}
\end{aligned}
$$

where $\overline{\boldsymbol{\Sigma}}$ is the deviatoric part of the macroscopic stress tensor $\boldsymbol{\Sigma}$ and $\overline{\boldsymbol{D}}$ is the deviatoric part of the macroscopic strain rate tensor. Let

$$
\Sigma_{p}=\frac{1}{3} \Sigma: \boldsymbol{X}
$$

with $\boldsymbol{X}$ defined in (11).

Combining the above two equations in (37), we obtain:

$$
3 \Sigma_{p}=\frac{\partial \Pi}{\partial B} \frac{1}{x_{2}} \quad \text { and } \quad \bar{\Sigma}=\frac{\partial \Pi}{\partial \boldsymbol{A}}
$$

It is recalled that $x_{2}=\frac{c^{3}}{a_{2} b_{2}{ }^{3}}$. Since $\Pi(\boldsymbol{D})$ is expressed in terms of $\widetilde{A}$ and $\widetilde{B}$, it is convenient to introduce in (39) the following change of variables $(\boldsymbol{A}, B \Rightarrow$ $\widetilde{A}, \widetilde{B})$ :

$$
\begin{aligned}
& 3 \Sigma_{p}=\frac{1}{x_{2}} \frac{\partial \Pi}{\partial \widetilde{B}} \frac{\partial \widetilde{B}}{\partial B}=\frac{p}{x_{2}} \frac{\partial \Pi}{\partial \widetilde{B}} \\
& \overline{\boldsymbol{\Sigma}}=\frac{\partial \Pi}{\partial \widetilde{A}} \frac{\partial \widetilde{A}}{\partial \boldsymbol{A}}+\frac{\partial \Pi}{\partial \widetilde{B}} \frac{\partial \widetilde{B}}{\partial \boldsymbol{A}}=\frac{\partial \Pi}{\partial \widetilde{A}} \frac{\partial \widetilde{A}}{\partial \boldsymbol{A}}-\frac{w}{p} \frac{\partial \Pi}{\partial \widetilde{B}} \mathbb{H}: \boldsymbol{Q}
\end{aligned}
$$

or:

$$
3 \Sigma_{p}=\frac{p}{x_{2}} \frac{\partial \Pi}{\partial \widetilde{B}} ; \quad \bar{\Sigma}+\frac{3 w x_{2}}{p^{2}} \Sigma_{p} \mathbb{H}: \boldsymbol{Q}=\frac{\partial \Pi}{\partial \widetilde{A}} \frac{\partial \widetilde{A}}{\partial \boldsymbol{A}}
$$

Let us introduce now the fourth order tensor $\mathbb{T}$ defined by:

$$
\mathbb{T}=\mathbb{J}+\mathbb{H}-\frac{3 w^{2} u_{1} u_{2}}{2 p^{2}}(\mathbb{H}: \boldsymbol{Q}) \otimes(\mathbb{H}: \boldsymbol{Q})
$$


Note that $\mathbb{T}$ is symmetric (has both major and minor symmetries). It follows that $\widetilde{A}$ can be written in the more compact form (see also (32)):

$$
\widetilde{A}=\sqrt{\frac{2}{3} \boldsymbol{A}: \mathbb{T}: \boldsymbol{A}}
$$

$\mathbb{T}$ being inversible, and taking into account the definitions (43) and (32), it is shown that the second relation in (41) can be expressed as:

$$
\frac{3}{2}\left(\bar{\Sigma}+\frac{3 w x_{2}}{p^{2}} \Sigma_{p} \mathbb{H}: \boldsymbol{Q}\right): \mathbb{T}^{-1}:\left(\bar{\Sigma}+\frac{3 w x_{2}}{p^{2}} \Sigma_{p} \mathbb{H}: \boldsymbol{Q}\right)=\left(\frac{\partial \Pi}{\partial \widetilde{A}}\right)^{2}
$$

for which the identity $\mathbb{J}: \boldsymbol{A}=0$ is used.

Because $\mathbb{T}$ is anisotropic, its inversion is a priori difficult. We seek $\mathbb{T}^{-1}$ in the following form:

$$
\mathbb{T}^{-1}=C_{1} \mathbb{J}+C_{2} \mathbb{M}+C_{3} \boldsymbol{Q} \otimes \boldsymbol{Q}
$$

where $C_{1}, C_{2}, C_{3}$ are three unknown constants and $\mathbb{M}$ is the fourth order tensor characterizing the anisotropy of the matrix (see (15)).

Using the identity $\boldsymbol{Q}: \mathbb{H}: \boldsymbol{Q}=\frac{2}{3} h_{2}$ and $\mathbb{T}: \mathbb{T}^{-1}=\mathbb{I}$, we obtain:

$$
C_{1} \mathbb{J}+C_{2} \mathbb{K}+\left[C_{3}-\frac{w^{2} u_{1} u_{2}}{2 p^{2}}\left(3 C_{2}+2 h_{2} C_{3}\right)\right](\mathbb{H}: \boldsymbol{Q}) \otimes \boldsymbol{Q}=\mathbb{I}
$$

where $h_{2}$ is given by (84). Therefore:

$$
C_{1}=1 ; \quad C_{2}:=1 ; \quad C_{3}=\frac{3}{2} \frac{w^{2} u_{1} u_{2}}{p^{2}-w^{2} u_{1} u_{2} h_{2}}
$$

Finally, using (44) together with (45) and (47), it is readily seen that:

$$
\left(\frac{\partial \Pi}{\partial \widetilde{A}}\right)^{2}=\Sigma_{e q}^{2}+\frac{1}{1-h_{2} \zeta}\left\{h_{2} \eta^{2} \Sigma_{p}^{2}+\zeta \Sigma_{q}^{2}+2 \eta \Sigma_{p} \Sigma_{q}\right\}
$$

where $\Sigma_{p}$ is given by Eq (38) while $\Sigma_{e q}$ denotes the equivalent stress according to Hill's criterion, i.e. $\Sigma_{e q}^{2}=\frac{3}{2} \Sigma: \mathbb{M}: \Sigma$.

$$
\Sigma_{q}=\Sigma: Q
$$


Let denote $\widetilde{\Sigma}_{e q}^{2}=\left(\frac{\partial \Pi}{\partial \widetilde{A}}\right)^{2}$ the generalized equivalent stress. The coefficients $\eta$ and $\zeta$ involved in the expression (48) of $\widetilde{\Sigma}_{e q}$ are given by:

$$
\eta=\frac{\kappa^{2} w x_{2}}{2} ; \quad \zeta=\frac{\kappa^{2} w^{2} u_{1} u_{2}}{4}
$$

Calculation of the coefficients $\kappa, \zeta$ and $\eta$ is given in Appendix (6.2). It is shown that $\kappa=\frac{3}{p}$ (see (98) for the expression of $p$ ) and that:

$$
\eta=\frac{\kappa^{2}(1+g)(f+g)\left(\alpha_{2}-\alpha_{1}\right)}{(1-f)} ; \quad \zeta=\frac{\kappa^{2}(1+g)(f+g)\left(\alpha_{2}-\alpha_{1}\right)^{2}}{(1-f)^{2}}
$$

\subsubsection{The closed form approximate expression of the macroscopic yield cri-} terion

Substituting the expression (44) of $\partial \widetilde{\Pi} / \partial \widetilde{A}$ and the expression (41) of $\partial \widetilde{\Pi} / \partial \widetilde{B}$ in equation (34), we obtain the general form of the yield criterion:

$$
\left(\frac{\widetilde{\Sigma}_{e q}}{\sigma_{0}}\right)^{2}+2(1+g)(f+g) \cosh \left\{\frac{\kappa \Sigma_{p}}{\sigma_{0}}\right\}-(1+g)^{2}-(f+g)^{2}=0
$$

where it is recalled that $\Sigma_{p}=\frac{1}{3} \boldsymbol{\Sigma}: \boldsymbol{X}, \Sigma_{q}=\boldsymbol{\Sigma}: \boldsymbol{Q}, \kappa=3 / p$ and $\widetilde{\Sigma}_{e q}$ is the generalized equivalent stress given by (48). The constant $g$ involved in the expression of the yield criterion is a shape parameter. It is equal to 0 for a prolate void while for an oblate void:

$$
g=\rho x_{2}=\rho \frac{c^{3}}{a_{2} b_{2}^{2}}=\rho \frac{e_{2}^{3}}{\sqrt{1-e_{2}^{2}}}
$$

Let us recall that the definition of coefficient $\rho$ is given in Appendix 6.2. Equation (52), together with (48), (51), (98) and (53) constitute the most important result of this study. It defines in the general case of arbitrary loadings the macroscopic yield surface of a porous anisotropic material containing prolate or oblate voids.

It is worth noting that the new criterion accounts for the coupling between the plastic anisotropy of the matrix and the void induced anisotropy through the fourth-order tensor $\mathbb{T}$ (see (42)) which is involved in the expression of the generalized equivalent stress $\widetilde{\Sigma}_{e q}(48)$. More precisely, if the void is not oriented along an axis of symmetry of the matrix, then the overall plastic behavior is characterized by an anisotropy more pronounced than that of the matrix ${ }^{4}$.

\footnotetext{
$\overline{4}$ The effects of the interaction between matrix anisotropy and void-shape induced anisotropy has been studied using a purely phenomenological approach by [11].
} 
Obviously, this modification of the plastic symmetry due to the void orientation will strongly affects the macroscopic behavior of the porous medium.

Let us also note that since $\mathbb{T}$ has both major and minor symmetries, the yield surface (52) is convex.

\section{Validation of the approximate yield criterion}

We will further assess the accuracy of the proposed criterion (52). The validations that we will perform are of two different types. First, we will show that for the special cases of spherical or cylindrical voids in a plastic anisotropic matrix and that of prolate or oblate voids in an isotropic matrix, respectively the new yield criterion reduces to existing approximate yield criteria.

To validate the approximations made in the analytic evaluation of the macroscopic plastic dissipation $\Pi(\boldsymbol{D})$, we will also present comparisons between the derived approximate criterion and the one obtained by numerical calculations of $\Pi(\boldsymbol{D})$.

\subsection{Case of spherical voids and of cylindrical cavity in an orthotropic matrix}

For the hollow sphere, which is a particular case of a prolate void or an oblate void $\left(e_{1} \rightarrow 0\right.$ and $\left.e_{2} \rightarrow 0\right)$, it is easy to check that $g=0, \alpha_{1}=\alpha_{2}=\frac{1}{3}$, $\beta_{1}=\beta_{2}=\frac{2}{5}, \eta=\zeta=0$ so $\widetilde{\Sigma}_{e q}^{2}=\Sigma_{e q}^{2}$ while $\Sigma_{p}=\Sigma_{h}$. Thus, the proposed criterion (52) reduces to:

$$
\begin{aligned}
& \left(\frac{\Sigma_{e q}}{\sigma_{0}}\right)^{2}+2 f \cosh \left\{\frac{\kappa \Sigma_{h}}{\sigma_{0}}\right\}-1-f^{2}=0 \\
& \text { with }: \quad \kappa=\frac{3}{p}=\frac{3}{2} \sqrt{\frac{5}{2 h_{1}+h_{2}+2 h_{3}}}
\end{aligned}
$$

which is Benzerga and Besson [2] approximate yield criterion for porous solids made of an orthotropic matrix obeying Hill's [19] plastic criterion and containing spherical voids.

For cylindrical voids $\left(e_{1} \rightarrow 1\right.$ and $\left.e_{2} \rightarrow 1\right), \eta=\zeta=0$, so $\widetilde{\Sigma}_{e q}^{2}=\Sigma_{e q}^{2}$ and $\Sigma_{p}=\left(\Sigma_{11}+\Sigma_{22}\right) / 2$ (see Table 1 for the values of $\alpha$ and $\beta$ ). Thus, for cylindrical cavities, the proposed criterion takes the form:

$$
\left(\frac{\Sigma_{e q}}{\sigma_{0}}\right)^{2}+2 f \cosh \left\{\frac{\sqrt{3}}{2 \sqrt{h_{1}}} \frac{\left(\Sigma_{11}+\Sigma_{22}\right)}{\sigma_{0}}\right\}-1-f^{2}=0
$$

i.e. reduces to Benzerga and Besson [2] approximate yield criterion for orthotropic material with cylindrical voids. 
Another case of interest is that of sheet metals. As already mentioned, Liao et al. [26] has proposed an analytic anisotropic Gurson-like yield criterion based on a limit analysis of a circular thin disk with a unique through-thickness void subjected to axisymmetric loading. The matrix was assumed to be rigid perfectly plastic, incompressible, and obeying Hill's [19] quadratic anisotropic criterion. Furthermore, it was assumed that the anisotropy in the plane of the disk is small i.e. that the Lankford coefficient $r_{\theta}$ in any direction $\theta$ in the plane of the sheet has the same value $R\left(r_{\theta}\right.$ is defined as the ratio of the in-plane transverse plastic strain to the through thickness plastic strain for uniaxial loading along a direction at an angle $\theta$ with the rolling direction). It can be easily verified that in this case, the anisotropy of the matrix is described by a single anisotropy coefficient $R$, i.e.:

$$
H=\frac{2}{3} \frac{R}{1+R} ; \quad F=G=\frac{2}{3} \frac{1}{1+R} ; \quad N=\frac{2}{3} \frac{1+2 R}{1+R}
$$

so $h_{1}$ (see equation (84)) is given by:

$$
h_{1}=\frac{3(1+R)}{2(1+2 R)}
$$

For the case of a thin sheet with cylindrical voids, the proposed criterion (52) is of the form (55) with $h_{1}$ given by (57), i.e.:

$$
\left(\frac{\Sigma_{e q}}{\sigma_{0}}\right)^{2}+2 f \cosh \left\{\sqrt{\frac{1+2 R}{2(1+R)}} \frac{\Sigma_{11}+\Sigma_{22}}{\sigma_{0}}\right\}-1-f^{2}=0
$$

which is identical to the yield criterion proposed by Liao et al. [26]. We refer the reader interested in numerical validations of (58) to the recent studies of Liao [27] and Chien et al. [9].

4.2 Prolate and oblate voids in an isotropic (von Mises) matrix: the case of axisymmetric loadings

In the following, we consider prolate and oblate cavities whose axes of symmetry coincide with axes of orthotropy of the matrix.

\subsubsection{Introductory comments}

Let us first recall the results established by [14] and [15] (see also [17]) for the case of a porous solid made of an isotropic matrix obeying von Mises yield 
criterion containing spheroidal voids and subjected to axisymmetric loading i.e. $\boldsymbol{\Sigma}=\Sigma_{11}\left(\underline{e}_{1} \otimes \underline{e}_{1}+\underline{e}_{2} \otimes \underline{e}_{2}\right)+\Sigma_{33} \underline{e}_{3} \otimes \underline{e}_{3}$. In our notations, the approximate yield criteria proposed by these authors are expressed as:

$$
\begin{gathered}
\frac{1}{1-\zeta_{G}} \frac{\left(\Sigma_{33}-\Sigma_{11}+\eta_{G} \Sigma_{p}\right)^{2}}{\sigma_{0}^{2}}+2(1+g)(f+g) \cosh \left\{\frac{\kappa_{G} \Sigma_{p}}{\sigma_{0}}\right\} \\
-(1+g)^{2}-(f+g)^{2}=0
\end{gathered}
$$

where the coefficients $\eta_{G}, \zeta_{G}$ and $\kappa_{G}$ will be specified in subsections 4.2 .2 and 4.2.3.

For the case of an isotropic matrix $h_{1}=h_{2}=h_{3}=1$; for axisymmetric loading $\Sigma_{e q}=\left|\Sigma_{33}-\Sigma_{11}\right|$ so the proposed criterion (52) reduces to a form similar to (59):

$$
\begin{gathered}
\frac{1}{1-\zeta} \frac{\left(\Sigma_{33}-\Sigma_{11}+\eta \Sigma_{p}\right)^{2}}{\sigma_{0}^{2}}+2(1+g)(f+g) \cosh \left\{\frac{\kappa \Sigma_{p}}{\sigma_{0}}\right\} \\
-(1+g)^{2}-(f+g)^{2}=0
\end{gathered}
$$

where $\zeta$ and $\eta$ are given, for a prolate or an oblate void in a von Mises matrix, by (51) and $\kappa$ by:

$$
\kappa=\left[\frac{1}{3} \frac{(1+g)(f+g)}{f(1-f)}\left\{\left(1+3 \alpha_{1}\right)\left(1-\alpha_{1}\right)-f\left(1+3 \alpha_{2}\right)\left(1-\alpha_{2}\right)\right\}\right]^{-1}
$$

Therefore, the comparison between the proposed criterion and the one proposed by Gologanu and coworkers [14];[15] reduces to a comparison between the approximations for the coefficients $\kappa, \eta$ and $\zeta$, respectively. These comparisons will be presented in the following for both prolate and oblate voids.

\subsubsection{Prolate voids in a von Mises matrix}

Two approximate expressions for $\kappa_{G}$ have been proposed by Gologanu et al. (see [14] and [15]):

$$
\begin{gathered}
\kappa_{G, 1 s t \text { app }}=\left[\frac{1}{\sqrt{3}}+(\sqrt{3}-2) \frac{\ln \left(e_{1} / e_{2}\right)}{\ln (f)}\right]^{-1} \\
\kappa_{G, 2 n d \text { app }}=\left[\frac{1}{\sqrt{3}}+(\sqrt{3}-2) \frac{\ln \left(e_{1} / e_{2}\right)}{\ln (f)}-\left(\frac{2}{\sqrt{3}}-1\right) \frac{e_{1}^{2}-e_{2}^{2}}{\ln (f)}\right]^{-1}
\end{gathered}
$$


For a fixed porosity, $f=0.3$, in Figure 5 are shown $\kappa_{G, 1 s t \text { app }}, \kappa_{G, 2 n d \text { app }}$, the proposed approximation of $\kappa$ as function of the eccentricity $e_{1}$ in comparison with the exact $\kappa$ (see (96) in the Appendix). It is observed that, although $\kappa_{2 n d}$ app. is close to the exact solution, the proposed approximation (61) is in better agreement.

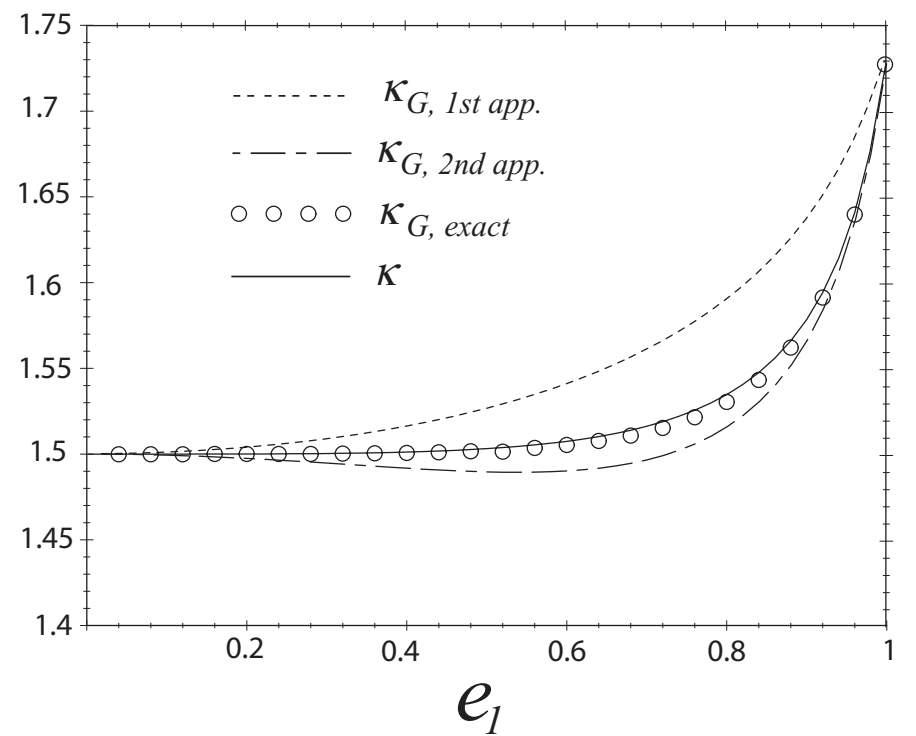

Fig. 5. Comparison of different approximations of $\kappa$ as function of $e_{1}$ in the case of a prolate cavity with a porosity $f=0.3$.

Note that in the case of a prolate void, Gologanu et al., [14] have neglected the "crossed" terms, so $\eta_{G}=\zeta_{G}=0$. Subsequently, these authors proposed in [17] certain expressions for $\eta_{G}$ and $\zeta_{G}$ such that for purely deviatoric loading $\left(D_{h}=0\right)$, the approximate solution coincides with the exact solution. These expressions are:

$$
\begin{aligned}
& \eta_{G}=\frac{\kappa_{G}(1-f) f \sinh (K)}{1+f^{2}+f[K \sinh (K)-2 \cosh (K)]} \\
& \zeta_{G}=1-\eta_{G} \frac{(1-f) \kappa_{G}-\eta_{G} K}{f \kappa_{G}^{2} \sinh (K)} \\
& \text { with }: \quad K=\kappa_{G}\left(\alpha_{2}-\alpha_{1}\right)
\end{aligned}
$$

Figures 6 and 7 show the variation of $\eta_{G}$ and $\zeta_{G}$ with the eccentricity $e_{1}$ according to the above equations (63) in which $\kappa_{G}$ is replaced by the exact solution i.e. $\kappa=\frac{3}{p}$ (see (96)). On the same figures are also depicted the proposed functions $\eta$ and $\zeta$ given by (51). A very good agreement is observed. This is not surprising, since $K$ is small and a development of the trigonometric term to the second order in (63) leads to the expressions (50) and consequently to the 
relations (51) for $\eta$ and $\zeta$ proposed in this study.

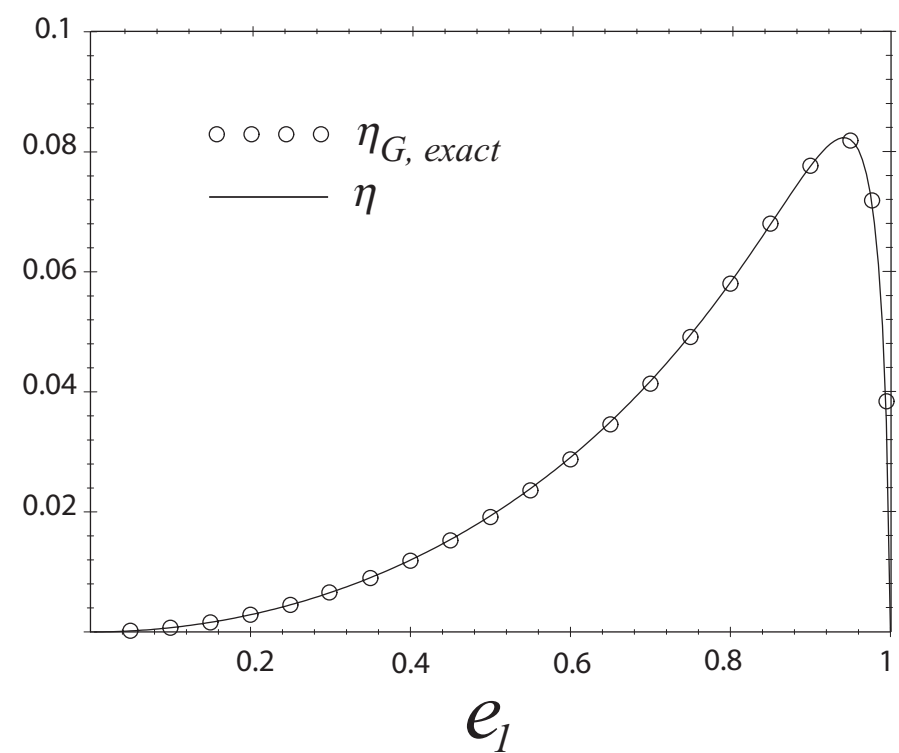

Fig. 6. Comparison of different approximations of $\eta$ as function of $e_{1}$ in the case of a prolate cavity with a porosity $f=0.3$.

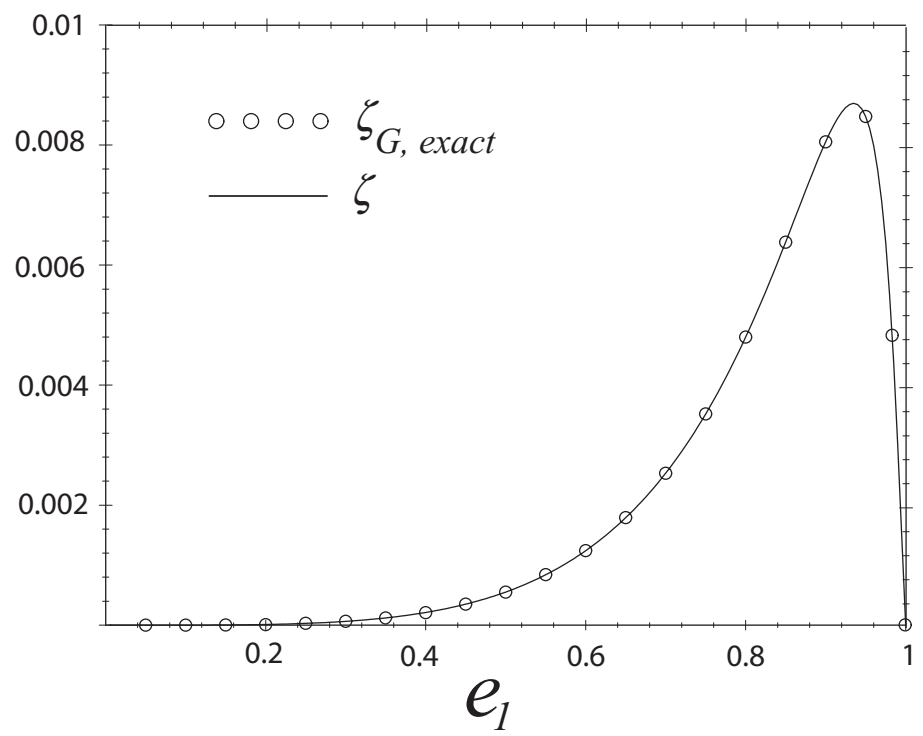

Fig. 7. Comparison of different approximations of $\zeta$ as function of $e_{1}$ in the case of a prolate cavity with a porosity $f=0.3$.

\subsubsection{Oblate voids in a von Mises matrix}

Let us first recall the approximation for $\kappa_{G}$ proposed by [15] in the case of oblate cavities. 


$$
\kappa_{G}=\left[\frac{2}{3}+\frac{g(1-f)(g+2 f+g f)}{3(g+1)^{2}(g+f)^{2} \ln \left(\frac{g+1}{g+f}\right)}\right]^{-1}
$$

For the case of oblate cavities, $e \rightarrow 1$, the crossed terms appearing in the integral expression of the macroscopic plastic dissipation cannot be neglected; thus $\eta$ and $\zeta$ are different from zero. In our notations, the expressions proposed by [15] for those coefficients are:

$$
\begin{aligned}
& \eta_{G}=\frac{\kappa_{G}(1-f)(g+1)(g+f) s h}{(g+1)^{2}+(g+f)^{2}+(g+1)(g+f)[K s h-2 c h]} \\
& \zeta_{G}=1-\eta_{G} \frac{(1-f) \kappa_{G}-\eta_{G} K}{(1+g)(g+f) \kappa_{G}^{2} \sinh (K)} \\
& \text { with }: \quad s h=\sinh (K) \text { and } \operatorname{ch}=\cosh (K)
\end{aligned}
$$

It is interesting to note that, by assuming $K$ sufficiently small and expanding the hyperbolic cosine and the hyperbolic sine to the second order ${ }^{5}$, we obtain an expression for $\eta_{G}$ identical to the proposed expression for $\eta$ given by (51). As an example, in Figures 8, 9 and 10 are shown comparisons of these approximations of $\eta$ and $\zeta$ for an oblate geometry and a porosity $f=0.3$. Again, a good agreement between the proposed approximations and the exact values is observed.

The approximate criterion proposed by Gologanu et al [15] for oblate cavities involves also a coefficient $C$. In the proposed formulation, the equivalent of this coefficient $C$ is the term $\frac{1}{1-\zeta}$. However, we note that $C$ is very close to 1 when $f \ll 1$ and $g \ll 1$. Indeed for these situations, it can be shown that $\zeta$, defined in the present paper by (50) and (99) tends to 0 . Thus, this coefficient has little influence on the accuracy of the criterion. However, it is non-negligible when $g \rightarrow+\infty$. This special case corresponds to a "sandwich" geometry i.e. a cavity of infinite dimensions in the directions $\underline{e}_{1}$ and $\underline{e}_{2}$ which has zero volume.

Based on the results presented, it can be concluded that, for the particular case of a porous medium made up of a von Mises matrix and spheroidal voids, the proposed criterion agrees well with the results of Gologanu et al. [14], [15] for axisymmetric loading.

$\overline{5}$ This solution was applied in the case of prolate cavities by [17], in order to take into account the crossed terms. 


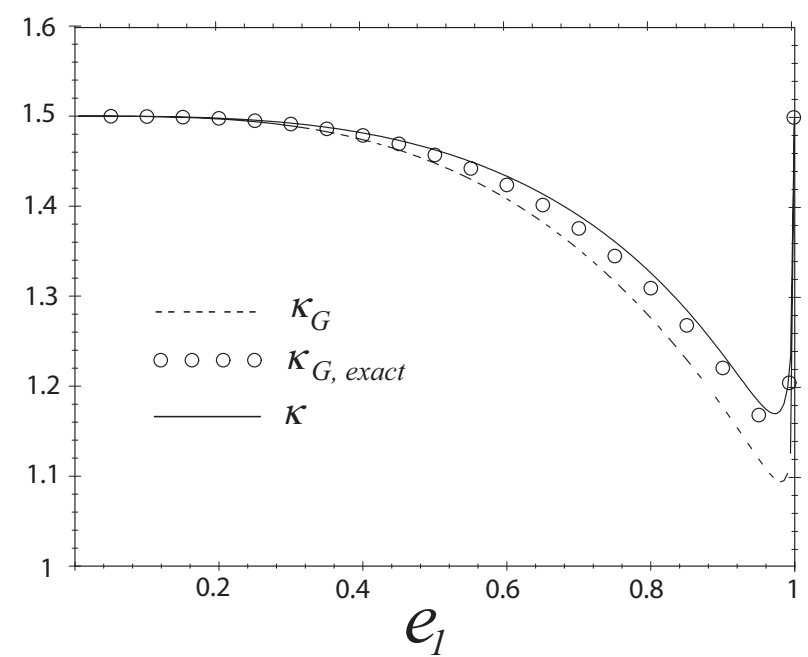

Fig. 8. Comparison of different approximations of $\kappa$ as function of $e_{1}$ in the case of an oblate cavity and porosity $f=0.3$.

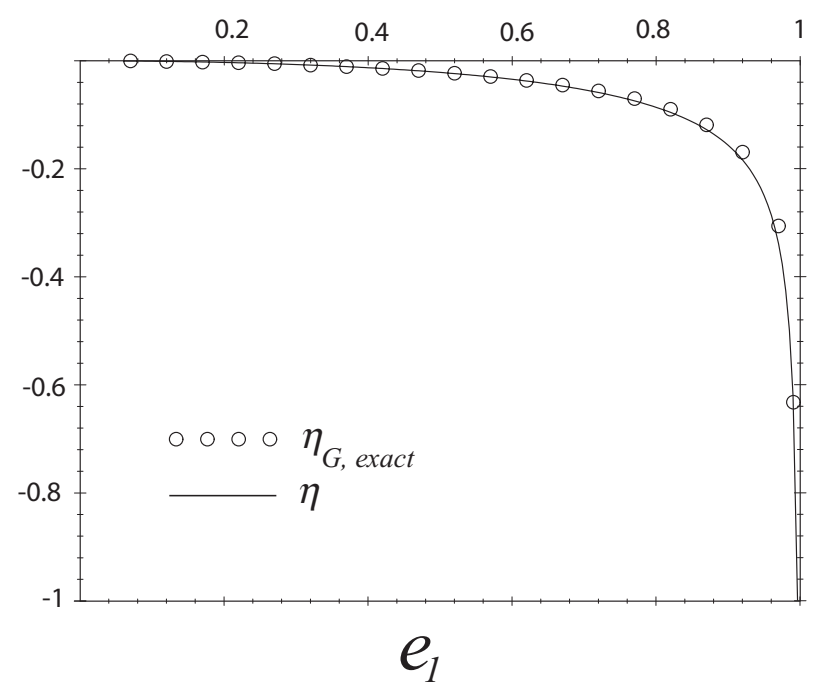

Fig. 9. Comparison of different approximations of $\eta$ as function of $e_{1}$ in the case of an oblate cavity and porosity $f=0.3$.

4.3 Prolate and oblate voids in an isotropic (von Mises) matrix: generalization to arbitrary loadings

We examine now the case when the porous solid is subjected to arbitrary loadings. Again, we assume that the considered porous material is still made up of an isotropic matrix i.e. we set $h_{2}=1$ in $\widetilde{\Sigma}_{e q}^{2}$ (equation (48)) which is required in the expression (52) of the proposed criterion. For comparison purposes, it is convenient to rewrite (52) in the following form: 


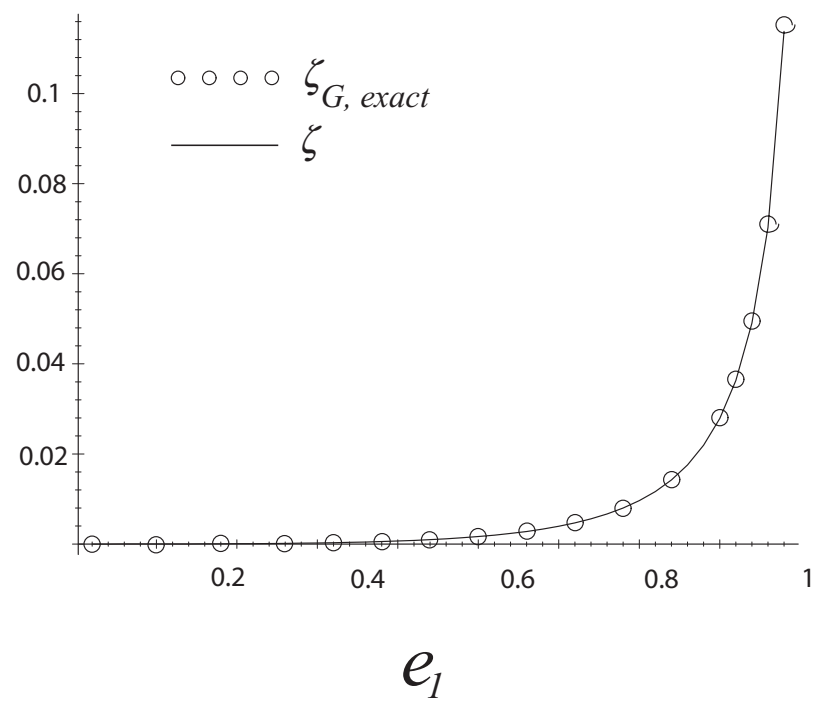

Fig. 10. Comparison of different approximations of $\zeta$ as function of $e_{1}$ in the case of an oblate cavity and porosity $f=0.3$.

$$
\begin{gathered}
\frac{C}{\sigma_{0}^{2}}\left\|\boldsymbol{\Sigma}^{\prime}+\eta \Sigma_{h} \boldsymbol{X}\right\|^{2}+2 \\
+1+g)(f+g) \cosh \left\{\kappa \frac{\Sigma_{h}}{\sigma_{0}}\right\}-(1+g)^{2}-(f+g)^{2} \\
+\frac{1-C}{\sigma_{0}^{2}}\left(\left\|\boldsymbol{\Sigma}^{\prime}\right\|^{2}-\Sigma_{q}^{2}\right)=0
\end{gathered}
$$

with $C=\frac{1}{1-\zeta}$. In the above equation (66), $\|$.$\| stands for the von Mises$ norm: $\|\boldsymbol{T}\|=\left(\frac{3}{2} \boldsymbol{T}: \boldsymbol{T}\right)^{\frac{1}{2}}$.

Next, we compare this expression with existing results proposed in the literature. We begin with a comparison of our criterion with the one proposed by Gologanu et al [15] for oblate cavities and by Gologanu [17] for prolate cavities. These authors have developed an approximate yield criteria based on a Gurson-like analysis for the case of axisymmetric loading and further proposed heuristical generalizations of these criteria to arbitrary loadings.

$$
\begin{gathered}
\frac{C}{\sigma_{0}^{2}}\left\|\Sigma^{\prime}+\eta \Sigma_{h} \boldsymbol{X}\right\|^{2}+2(1+g)(f+g) \cosh \left\{\kappa \frac{\Sigma_{h}}{\sigma_{0}}\right\}-(1+g)^{2} \\
-(f+g)^{2}=0
\end{gathered}
$$

Comparison of the above equation with expression (66) derived in the present study shows that $(67)$ does not contain the term $\frac{1-C}{\sigma_{0}^{2}}\left(\left\|\Sigma^{\prime}\right\|^{2}-\Sigma_{q}^{2}\right)$. Note that, since in various cases, $\zeta$ is small, and so $C \rightarrow 1$, this term can be neglected.

A slightly more elaborate generalization to arbitrary loadings was proposed by Gologanu [16] on the basis of a micromechanical analysis. This criterion reads: 


$$
\begin{gathered}
\frac{C}{\sigma_{0}^{2}}\left\|\boldsymbol{\Sigma}^{\prime}+\eta \Sigma_{h} \boldsymbol{X}\right\|^{2}+2(1+g)(f+g) \cosh \left\{\kappa \frac{\Sigma_{h}}{\sigma_{0}}\right\}-(1+g)^{2}-(f+g)^{2} \\
+\frac{1-C}{\sigma_{0}^{2}}\left(\left\|\boldsymbol{\Sigma}^{\prime}\right\|^{2}+3 \Sigma_{x z}^{2}+3 \Sigma_{y z}^{2}\right)=0
\end{gathered}
$$

Comparison between Gologanu [16] criterion and the proposed one (66) shows that the expression (68) reported in [16] still is different: the last parenthesis of (68) does not coincide with the one of (66).

\subsection{Prolate and oblate voids in a Hill-type matrix: axisymmetric loading}

The proposed anisotropic criterion (52) was obtained by making certain assumptions that allowed us to obtain an analytical expression of the macroscopic plastic dissipation. We will assess the accuracy of the proposed criterion by comparing the analytical yield loci to "exact" yield loci obtained by evaluating numerically the same integral (given by (26)). Figures 11 to 12 show the theoretical yield loci (full line) for low porosity $f=0.01$ and moderate porosity $f=0.1$ in comparison with the numerical ones (symbols) for prolate cavities with $a_{1} / b_{1}=2$. Similarly, on Figures 13 and 14 are shown comparisons between theoretical and numerical results for an oblate cavity with $b_{1} / a_{1}=5$. The numerical values for Hill's anisotropy coefficients are: $M_{11}=0.733, M_{22}=0.57, M_{33}=0.499, M_{44}=3.669, M_{55}=1.141, M_{66}=2.2$. These values correspond to a steel (experimental data from Benzerga [1]).

Note an overall good agreement between the approximate criteria (full line) and the exact two fields velocity-based criterion (discrete points). This confirms in the general case of an anisotropic matrix the excellent quality of the approximations considered in this work.

For illustration purposes, the anisotropic yield surface obtained in the case of an anisotropic Hill matrix is compared in figures 11 to 16 with that corresponding to a von Mises matrix. This comparison clearly shows the effects associated to the plastic anisotropy of the matrix. Complete validation of the proposed yield criteria is beyond the scope of the present paper. Further validation of the proposed criterion based on finite element cell calculations will be considered in future research.

\subsubsection{Penny-shaped cracks}

In the case of penny-shaped (circular) cracks (i.e. $f=0$ and oblate cavities with $e_{1} \rightarrow 1, e_{2}$ arbitrary), the proposed criterion (52) reads:

$$
\frac{\tilde{\Sigma}_{e q}^{2}}{\sigma_{0}^{2}}+2 \rho d(1+\rho d) \cosh \left\{\frac{\kappa \Sigma_{p}}{\sigma_{0}}\right\}-(1+\rho d)^{2}-\rho^{2} d^{2}=0
$$




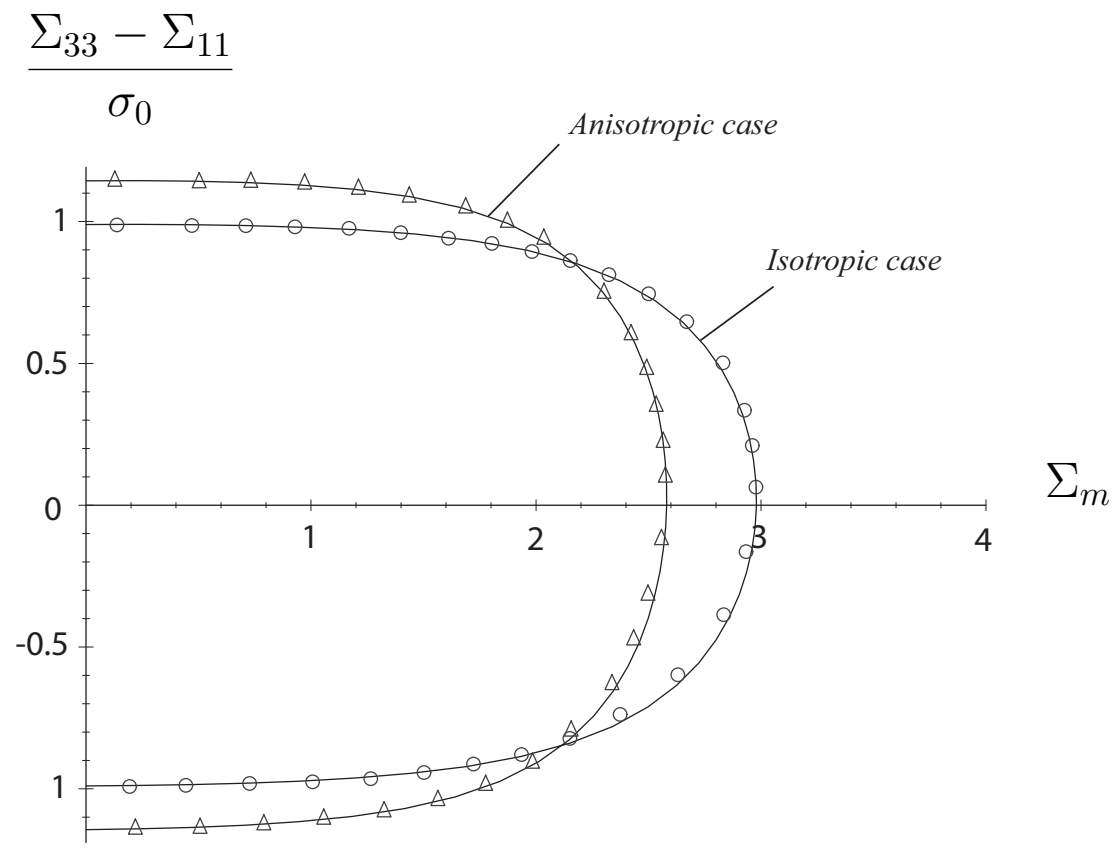

Fig. 11. Comparison between isotropic and anisotropic yield locus for a prolate cavity, with $a_{1} / b_{1}=2$, porosity $f=0.01$.

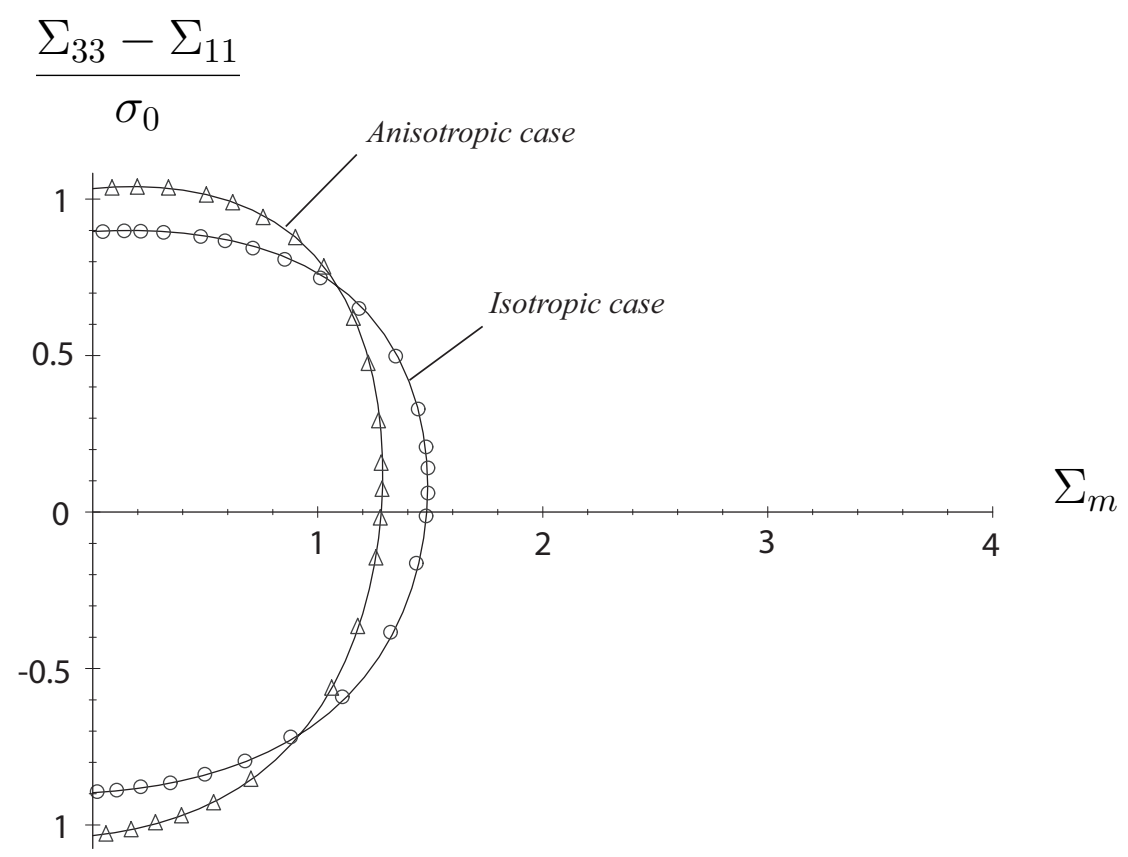

Fig. 12. Comparison between isotropic and anisotropic yield locus for a prolate cavity, with $a_{1} / b_{1}=2$, porosity $f=0.1$. 


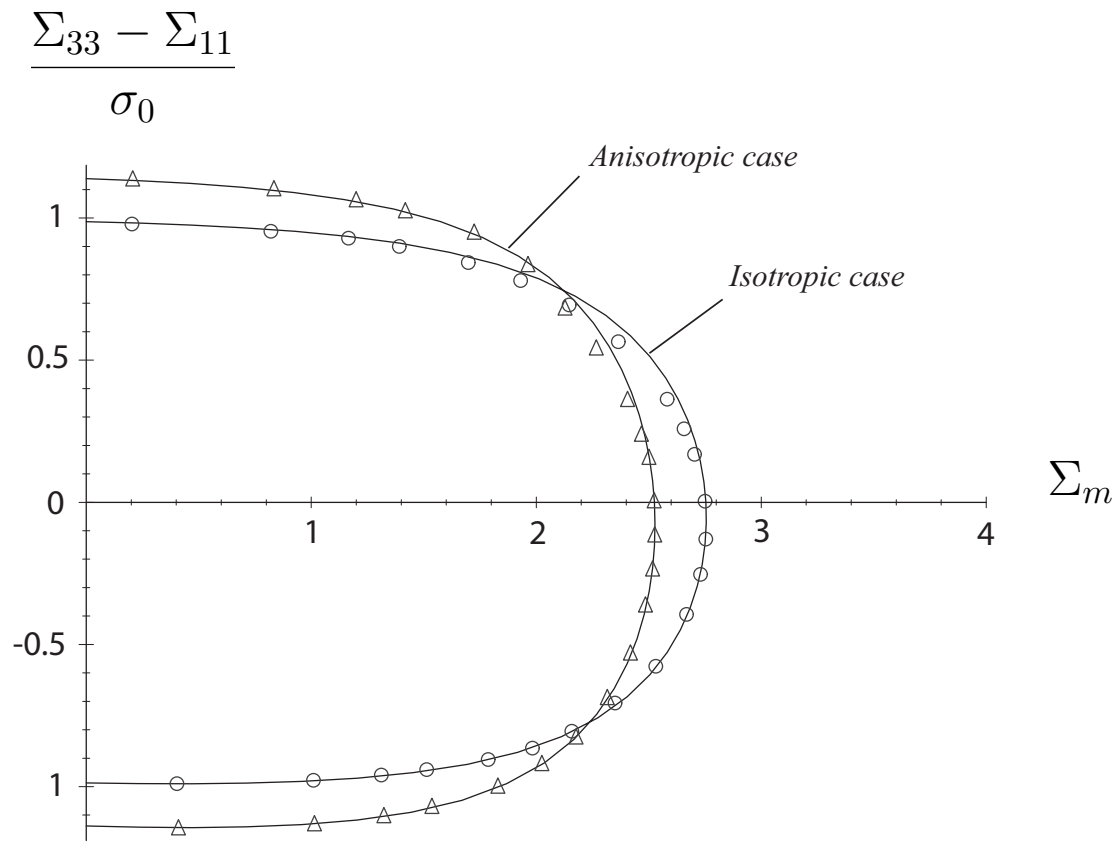

Fig. 13. Comparison between isotropic and anisotropic yield locus for an oblate cavity, with $b_{1} / a_{1}=5$, porosity $f=0.01$.

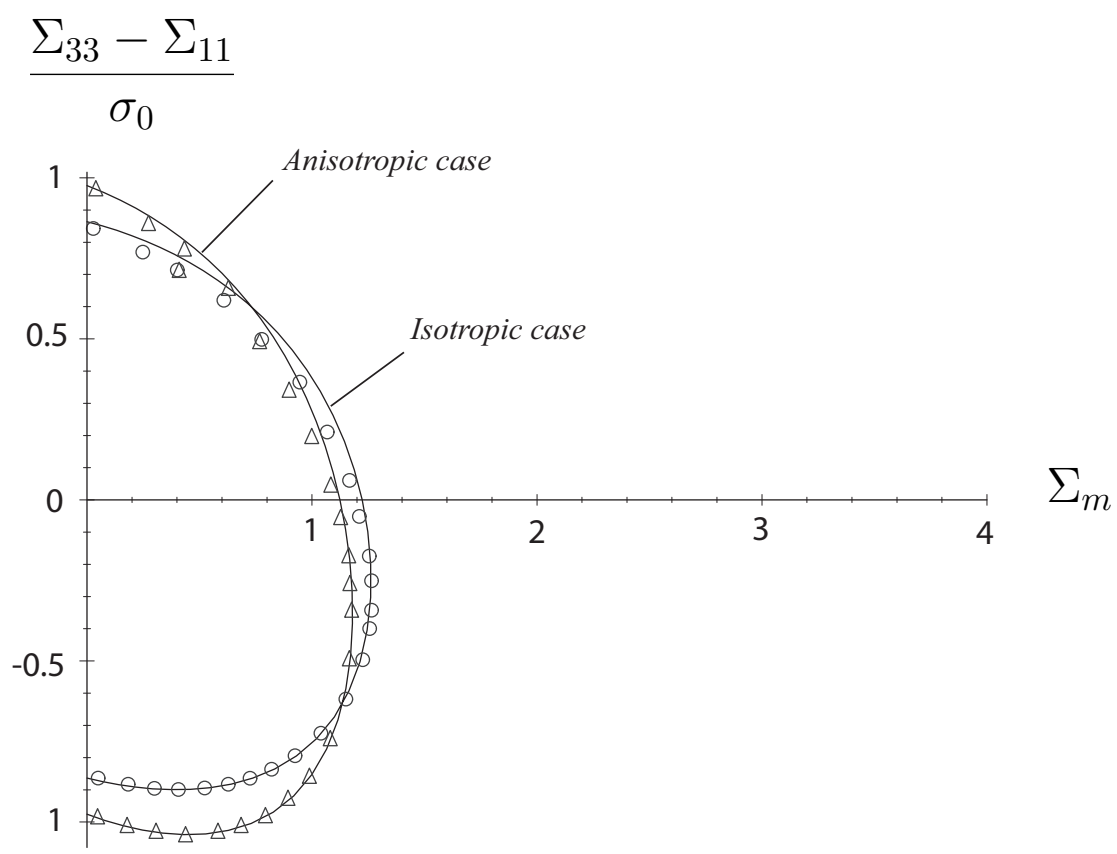

Fig. 14. Comparison between isotropic and anisotropic yield locus for an oblate cavity, with $b_{1} / a_{1}=5$, porosity $f=0.1$. 
where $g=\rho d$ has been used. Coefficient $\rho$ is a function of the anisotropy coefficients given in appendix (section 6.2).

The coefficients $\eta, \zeta$ and $\kappa$ involved in the expression of the generalized equivalent stress $\widetilde{\Sigma}_{e q}$ (see eqs (48)) are:

$$
\begin{aligned}
& \eta=\kappa^{2}\left(\alpha_{2}-1\right)(1+\rho d) \rho d \quad \zeta=\kappa^{2}\left(\alpha_{2}-1\right)^{2}(1+\rho d) \rho d \\
& \kappa=3\left[\frac { 3 } { 2 } ( 1 + \rho d ) \rho \left[\frac{\pi}{2}\left(h_{1}+3 h_{2}+4 h_{3}\right)\right.\right. \\
& \left.\left.+d\left[\beta_{2}\left(h_{1}+3 h_{2}-4 h_{3}\right)+6 \alpha_{2}\left(1-\alpha_{2}\right) h_{2}+\left(1-\alpha_{2}\right)\left(h_{1}-3 h_{2}+4 h_{3}\right)\right)\right]\right]^{-1 / 2}
\end{aligned}
$$

In the above equations, $\alpha_{2}$ and $\beta_{2}$ are functions of the eccentricity $e_{2}, g=\rho d$, where $d$ is Budiansky and O'Connel's [8] crack density parameter:

$$
d=\frac{e_{2}^{3}}{\sqrt{1-e_{1}^{2}}}=\frac{b_{1}^{3}}{a_{2} b_{2}^{2}}=\frac{\frac{4 \pi}{3} b_{1}^{3}}{|\Omega|}
$$

in which $|\Omega|$ denotes the volume of the studied cell.

On figures 15 and 16 are shown comparison between theoretical and numerical results for different values of the crack density parameter: $d=0.01, d=0.1$ and $d=0.5$. In the anisotropic case the Hill's coefficients are the same as the ones used in the previous section. Still again, comparison clearly shows the effects related to the plastic anisotropy of the matrix.

\section{Conclusions}

New yield criteria for porous ductile materials which account simultaneously for the effects of plastic anisotropy of the solid matrix and the voids shape have been developed based on a limit-load analysis. The matrix was assumed to obey Hill's quadratic yield criterion, while the voids were considered to be ellipsoidal of either prolate or oblate shape. The new approximate criteria are valid for the general case of arbitrary loadings. The effects of matrix anisotropy on yielding of voided materials were illustrated by comparing the yield loci corresponding to a Hill matrix and either oblate or prolate cavities with that corresponding to a Mises matrix. However, in order to assess the effect of matrix anisotropy on void growth and how the presence of voids affect the overall anisotropy finite element simulations need to be performed. Nevertheless, based on the qualitative trends shown in this paper one may expect that the present model may have a large spectrum of applications. For example, the incorporation of the proposed models into a multi-scale model for high-cycle fatigue can be found in Monchiet [29]. It is worthwhile noting 


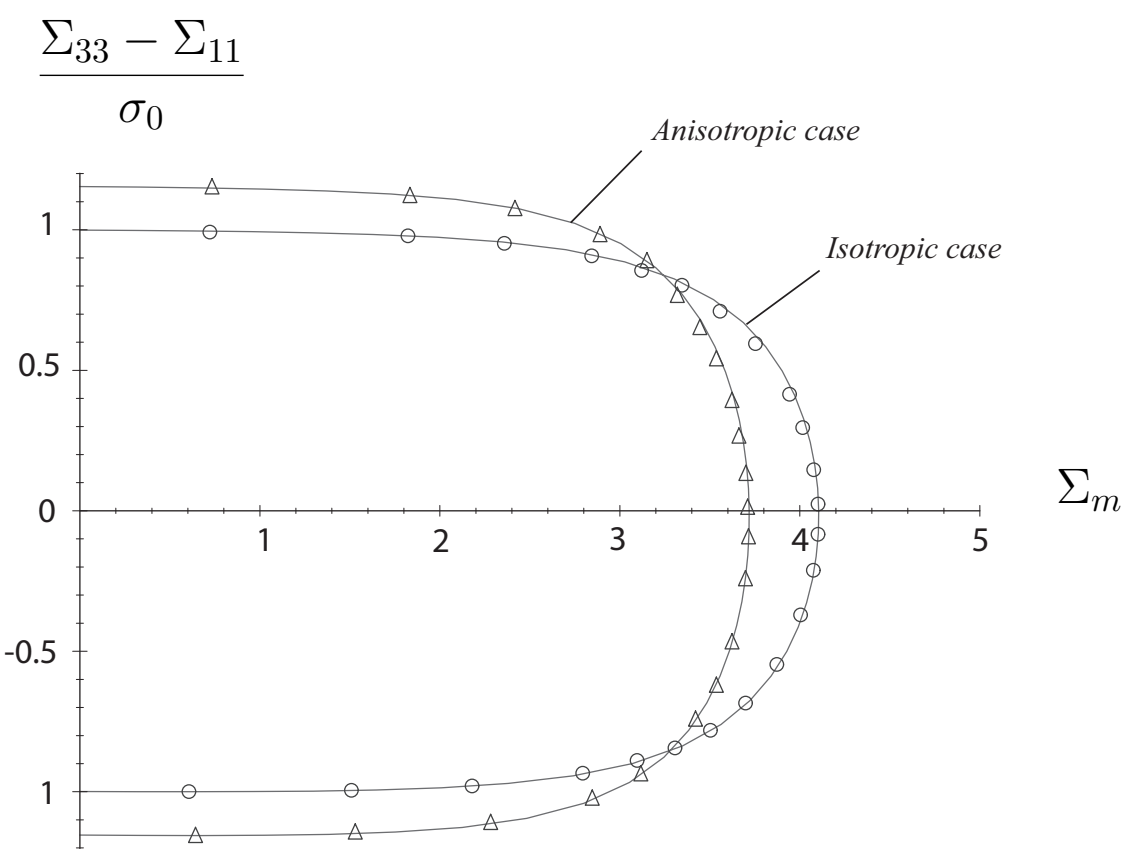

Fig. 15. Comparison between isotropic and anisotropic yield locus for a penny-shaped crack with cracks density parameter $d=0.01$.

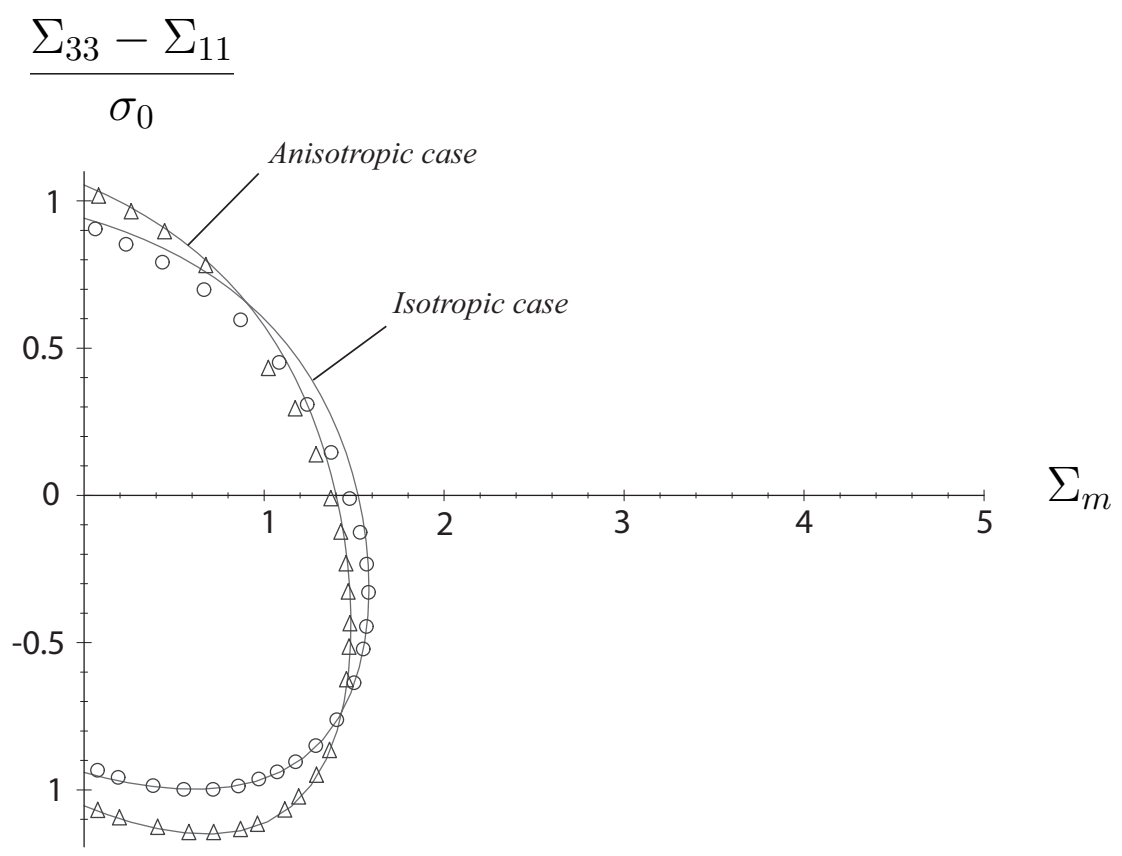

Fig. 16. Comparison between isotropic and anisotropic yield locus for a penny-shaped crack with cracks density parameter $d=0.5$.

that it was proposed a unique procedure for obtaining the approximate yield criteria for either prolate or oblate voids. It was shown that for specific cases, the proposed criteria reduce to existing Gurson-type models. For the case of a Von Mises matrix and axisymmetric loading, the proposed criterion reduces to that of Gologanu and co-workers [14], [15], [17]. Furthermore, the criterion 
developed provides a rigorous micromechanics-based generalization of the [14], [15], [17] to the general case of arbitrary loading. Note that to date the only micromechanics-based attempts to generalize these yield criteria have been proposed by Gologanu [16] and Gologanu et al. [17]. However, the formulations given by these authors contain a minor misprint which was pointed out in this work.

In the case of cylindrical voids in a Hill type matrix, the proposed criterion reduces to the criterion of Liao and Pan [26] while in the case of spherical voids in a Hill matrix it reduces to the criterion proposed by Benzerga et al. [2]. The theoretical analysis in the case of arbitrary loadings, anisotropic matrix, and ellipsoidal voids is very complex. We determined a trial velocity field satisfying conditions of homogeneous boundary strain rate and used it to derive approximate yield criteria. It is worth noting that even using this velocity field, the resulting macroscopic plastic dissipation is very complicated and cannot be calculated analytically. A number of approximations were proposed in order to estimate it and thus provide analytic estimates of the overall yield criteria. To validate the proposed form of the yield criteria, we compared the proposed yield loci with that obtained by numerical evaluation of the plastic dissipation. A very good agreement was observed. However, for a complete validation of the proposed yield criteria, 3-D finite element calculations in which a larger family of trial velocity fields are considered need to be performed.

Finally, it must be emphasized that the formulation of a constitutive relation based on the proposed criterion can be done by following in a very closed manner the works by Gologanu et al. [17] or by Pardoen and Hutchinson [32]. As for the existing models in the field, this constitutive law derives solely from the incompressibility of the metallic matrix (Hill material) and the usual property of macroscopic normality. The use of such law in Finite Elements calculations will allow to achieve quantitative prediction of the role of plastic anisotropy in damage accumulation and fracture initiation in plastic structures.

\section{Appendix}

\subsection{Determination of the approximate expression of $\Pi(\boldsymbol{D})$ and description of the required approximations}

This subsection is devoted to the computation of $\Pi(\boldsymbol{D})$ starting from (29) with (30). It is convenient to express $\boldsymbol{d}^{E}$ (see (14)) in cylindrical coordinates:

$$
\begin{aligned}
& \boldsymbol{d}^{E}=\frac{3 c^{3}}{2 a b^{2}}(1-\alpha)\left(\mathbf{1}-3 \underline{e}_{\rho} \otimes \underline{e}_{\rho}\right) \\
& +\frac{3 c^{3}}{2 a b^{2}}\left[1-3 \alpha+\frac{2 b^{2} \cos ^{2}(\varphi)}{L_{\lambda}^{2}}\right]\left(\underline{e}_{\rho} \otimes \underline{e}_{\rho}-\underline{e}_{3} \otimes \underline{e}_{3}\right)-\frac{3 c^{3} \sin (2 \varphi)}{b L_{\lambda}^{2}} \underline{e}_{\rho} \stackrel{s}{\otimes} \underline{e}_{3}
\end{aligned}
$$


Since $\mathbb{H}: \mathbf{1}=0$, the cross term $\boldsymbol{A}: \mathbb{H}: \boldsymbol{d}^{E}$ is given by:

$$
\begin{aligned}
\boldsymbol{A}: \mathbb{H}: \boldsymbol{d}^{E} & =-\frac{9 c^{3}}{2 a b^{2}}(1-\alpha) \boldsymbol{A}: \mathbb{H}: \underline{e}_{\rho} \otimes \underline{e}_{\rho} \\
& +\frac{3 c^{3}}{2 a b^{2}}\left[1-3 \alpha+\frac{2 b^{2} \cos ^{2}(\varphi)}{L_{\lambda}^{2}}\right] \boldsymbol{A}: \mathbb{H}:\left(\underline{e}_{\rho} \otimes \underline{e}_{\rho}-\underline{e}_{3} \otimes \underline{e}_{3}\right) \\
& -\frac{3 c^{3} \sin (2 \varphi)}{b L_{\lambda}^{2}} \boldsymbol{A}: \mathbb{H}: \underline{e}_{\rho} \otimes \underline{e}_{3}
\end{aligned}
$$

while $d_{e q}^{E}=\frac{2}{3} \boldsymbol{d}^{E}: \boldsymbol{d}^{E}($ see $(25))$ is expressed as:

$$
\begin{aligned}
d_{e q}^{E^{2}}= & \frac{9 c^{6}}{4 a^{2} b^{4} L_{\lambda}^{4}}\left\{4 a^{4} \sin ^{4}(\varphi) H_{\rho \rho \rho \rho}+\left[(1-3 \alpha) L_{\lambda}^{2}+2 b^{2} \cos ^{2}(\varphi)\right]^{2} H_{3333}\right. \\
& +4\left[(1-3 \alpha) L_{\lambda}^{2}+2 b^{2} \cos ^{2}(\varphi)\right] a^{2} \sin ^{2}(\varphi) H_{\rho \rho 33} \\
& +4 a^{2} b^{2} \sin ^{2}(2 \varphi) H_{\rho 3 \rho 3}+8 a^{3} b \sin (2 \varphi) \sin ^{2}(\varphi) H_{\rho \rho \rho 3} \\
& \left.+4 a b \sin (2 \varphi)\left[(1-3 \alpha) L_{\lambda}^{2}+2 b^{2} \cos ^{2}(\varphi)\right] H_{\rho 333}\right\}
\end{aligned}
$$

Remark: in the particular case when the matrix is isotropic, i.e. $\mathbb{H}=\mathbb{K}$, for axisymmetric loading, we have:

$$
\begin{aligned}
& \boldsymbol{A}: \mathbb{H}: \underline{e}_{\rho} \otimes \underline{e}_{\rho}=-\frac{1}{2} A_{33} ; \quad \boldsymbol{A}: \mathbb{H}:\left(\underline{e}_{\rho} \otimes \underline{e}_{\rho}-\underline{e}_{3} \otimes \underline{e}_{3}\right)=-\frac{3}{2} A_{33} \\
& \boldsymbol{A}: \mathbb{H}: \underline{e}_{\rho} \otimes \underline{e}_{3}=0
\end{aligned}
$$

It follows that the crossed term $\boldsymbol{A}: \mathbb{H}: \boldsymbol{d}^{E}$ does not depend on the coordinate $\theta$. Furthermore, since the components of $\mathbb{H}$, in cylindrical coordinates are:

$$
H_{\rho \rho \rho \rho}=H_{3333}=\frac{2}{3} ; \quad H_{\rho \rho 33}=-\frac{1}{3} ; \quad H_{\rho 3 \rho 3}=\frac{1}{2} ; \quad H_{\rho \rho \rho 3}=H_{\rho 333}=0
$$

the expression of $d_{e q}^{E^{2}}$ is:

$$
d_{e q}^{E^{2}}=\frac{9 c^{6}}{2 a^{2} b^{4}}\left\{3(1-\alpha)^{2}-2(1-3 \alpha) \frac{a^{2} \sin ^{2}(\varphi)}{L_{\lambda}^{2}}\right\}
$$

It is readily seen from (77) that for an isotropic matrix and axisymmetric loading, $d_{e q}^{E}{ }^{2}$ does not depend on $\theta$. Moreover, the dependence of $d_{e q}$ on $\varphi$ is 
through $\cos ^{2}(\varphi)$. This has motivated the approximation proposed by [14-17] which consists in replacing $\cos ^{2}(\varphi)$ by $\frac{1}{3}$ which is its mean value over the interval $[0, \pi]$.

However, in the anisotropic case, the components of $\mathbb{H}$ in cylindrical coordinates depend on $\theta$. Thus, $d_{e q}^{E}$ and the "crossed" term $\boldsymbol{A}: \mathbb{H}: \boldsymbol{d}^{E}$ depend on $\theta$. This additional difficulty due to the matrix anisotropy led to the introduction of approximation $\mathcal{A} 1$. It is worth noting that for axisymmetric loading and matrix isotropy, $\mathcal{A} 1$ is identical to the approximation used by Gurson [18] and Gologanu et al. [14,15].

Let us now calculate the terms involved in the expression of $\left\langle d_{e q}^{2}>_{\mathcal{E}(\lambda)}\right.$. Let first note that since $\boldsymbol{A}$ is uniform in the matrix, $A_{e q}$ is a constant. Next, to calculate the mean value associated to the crossed term $\boldsymbol{A}: \mathbb{H}: \boldsymbol{d}^{E}$, we make use of the following identities:

$$
\frac{1}{2 \pi} \int_{\theta=0}^{\theta=2 \pi} \underline{e}_{\rho} \otimes \underline{e}_{\rho} d \theta=\frac{1}{2}\left(\mathbf{1}-\underline{e}_{3} \otimes \underline{e}_{3}\right) ; \quad \frac{1}{2 \pi} \int_{\theta=0}^{\theta=2 \pi} \underline{e}_{\rho} d \theta=0
$$

A straightforward but lengthy calculation leads to:

$$
\begin{aligned}
<\boldsymbol{A}: \mathbb{H}: & \boldsymbol{d}^{E}>_{\mathcal{E}}=\frac{3}{4 \pi\left(2 a^{2}+b^{2}\right)} \int_{\varphi=0}^{\varphi=\pi} \int_{\theta=0}^{\theta=2 \pi} \boldsymbol{A}: \mathbb{H}: \boldsymbol{d}^{E} L_{\lambda}^{2} \sin (\varphi) d \varphi d \theta \\
& =-\frac{9 c^{3} \boldsymbol{A}: \mathbb{H}: \boldsymbol{Q}}{2 a b^{2}\left(2 a^{2}+b^{2}\right)} \int_{\varphi=0}^{\varphi=\pi}\left[\alpha L_{\lambda}^{2}-b^{2} \cos ^{2}(\varphi)\right] \sin (\varphi) d \varphi \\
& =-\frac{3}{2} W(\lambda) \boldsymbol{A}: \mathbb{H}: \boldsymbol{Q}
\end{aligned}
$$

where $W(\lambda)$ is given by:

$$
W(\lambda)=\frac{2 c^{3}\left(a^{2}-b^{2}\right)}{a b^{2}\left(2 a^{2}+b^{2}\right)}(1-\alpha-\beta)
$$

while $\boldsymbol{Q}$ is the deviatoric second-order tensor defined as:

$$
\boldsymbol{Q}=\frac{1}{2}\left(\underline{e}_{1} \otimes \underline{e}_{1}+\underline{e}_{2} \otimes \underline{e}_{2}\right)-\underline{e}_{3} \otimes \underline{e}_{3}
$$

To calculate $<d_{e q}^{E^{2}}>_{\mathcal{E}(\lambda)}$, we first compute the mean value of $d_{e q}^{E^{2}}$ over $\theta \in[0,2 \pi]$. Since : 


$$
\begin{aligned}
& \frac{1}{2 \pi} \int_{0}^{2 \pi} H_{\rho \rho \rho \rho} d \theta=\frac{1}{4}\left\{H_{11}+H_{22}+\frac{1}{2} H_{33}+H_{66}\right\} \\
& \frac{1}{2 \pi} \int_{0}^{2 \pi} H_{\rho \rho \rho 3} d \theta=0 ; \quad \frac{1}{2 \pi} \int_{0}^{2 \pi} H_{\rho \rho 33} d \theta=-\frac{1}{2} H_{33} \\
& \frac{1}{2 \pi} \int_{0}^{2 \pi} H_{\rho 3 \rho 3} d \theta=\frac{1}{4}\left(H_{44}+H_{55}\right) ; \quad \frac{1}{2 \pi} \int_{0}^{2 \pi} H_{\rho 333} d \theta=0
\end{aligned}
$$

it follows that:

$$
\begin{aligned}
<d_{e q}^{E^{2}}>_{\theta}= & \frac{3 x^{2}}{L_{\lambda}^{4}}\left\{a^{4} \sin ^{4}(\varphi) h_{1}+3\left(b^{2} \cos ^{2}(\varphi)-\alpha L_{\lambda}^{2}\right)^{2} h_{2}\right. \\
& \left.+a^{2} b^{2} \sin ^{2}(2 \varphi) h_{3}\right\}
\end{aligned}
$$

where the parameters $h_{i}$ are given by ${ }^{6}$ :

$$
\begin{aligned}
h_{1} & =\frac{1}{4}\left(2 H_{11}+2 H_{22}+2 H_{66}-H_{33}\right) ; \quad h_{2}=\frac{3}{2} H_{33} ; \\
h_{3} & =\frac{1}{2}\left(H_{44}+H_{55}\right)
\end{aligned}
$$

Then, $<d_{e q}^{E^{2}}>_{\mathcal{E}(\lambda)}$ reads:

$$
\begin{aligned}
<d_{e q}^{E^{2}}>_{\mathcal{E}(\lambda)} & =\frac{9 x^{2}}{2 a^{2}+b^{2}}\left\{\left(a^{4} h_{1}+3 \alpha^{2} b^{2} h_{2}\right) I_{1}(\lambda)+3\left(b^{2}-\alpha a^{2}\right)^{2} h_{2} I_{2}(\lambda)\right. \\
+ & \left.2 b^{2}\left[2 a^{2} h_{3}-3\left(b^{2}-\alpha a^{2}\right) \alpha h_{2}\right] I_{3}(\lambda)\right\}
\end{aligned}
$$

where $I_{i}(\lambda)$ are given by:

$$
\begin{aligned}
& I_{1}(\lambda)=\frac{1}{2} \int_{0}^{\pi} \frac{\sin ^{5}(\varphi)}{L_{\lambda}^{2}} d \varphi=\frac{2}{3 a^{2}}(3-3 \alpha-\beta) \\
& I_{2}(\lambda)=\frac{1}{2} \int_{0}^{\pi} \frac{\cos ^{4}(\varphi) \sin (\varphi)}{L_{\lambda}^{2}}=\frac{2}{3 b^{2}}(1-\beta) \\
& I_{3}(\lambda)=\frac{1}{2} \int_{0}^{\pi} \frac{\cos ^{2}(\varphi) \sin ^{3}(\varphi)}{L_{\lambda}^{2}}=\frac{2}{3 a^{2}} \beta
\end{aligned}
$$

It follows that the mean value of $d_{e q}^{E}$ on each confocal spheroid reads:

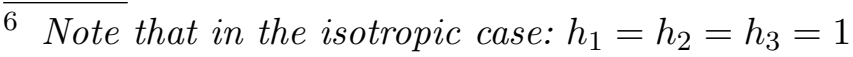




$$
<d_{e q}^{E^{2}}>_{\mathcal{E}(\lambda)}=P(\lambda)
$$

with

$$
\begin{aligned}
& P(\lambda)=\frac{3 x^{2}}{2 a^{2}+b^{2}}\left\{a^{2}(3-3 \alpha-\beta) h_{1}\right. \\
& \left.+3(1-\alpha-\beta)\left[a^{2} \alpha+b^{2}(1-\alpha)\right] h_{2}+4 b^{2} \beta h_{3}\right\}
\end{aligned}
$$

Note that $P(\lambda)$ depends explicitly on the anisotropy coefficients $h_{i}$.

Substituting (79) and (87) in (30), we obtain the mean value of $<d_{e q}^{2}>_{\mathcal{E}(\lambda)}$ on each confocal spheroid:

$$
<d_{e q}^{2}>_{\mathcal{E}(\lambda)}=A_{e q}^{2}-2 W(\lambda) B \boldsymbol{A}: \mathbb{H}: \boldsymbol{Q}+P(\lambda) B^{2}
$$

Further approximations need to be considered in order to compute the plastic dissipation $\Pi(\boldsymbol{D})$ i.e. to evaluate $\int_{x_{1}}^{x_{2}}\left\{<d_{e q}^{2}>_{\mathcal{E}(\lambda)}\right\}^{1 / 2} \frac{d x}{x^{2}}$ (see (29)).

Note that the spherical void is the particular case of a prolate void for which $e_{1} \rightarrow 0, e_{2} \rightarrow 0$. Thus $e \rightarrow 0$ or equivalently $\lambda \rightarrow 0$. It follows that (see (87)):

$$
P(\lambda) \rightarrow \frac{4}{5} x^{2}\left(2 h_{1}+h_{2}+2 h_{3}\right)
$$

where $x=c^{3} /\left(a b^{2}\right)$.

For a cylindrical void, $e \rightarrow 1$ or equivalently $\lambda \rightarrow+\infty$ and $P(\lambda)$ takes the following value:

$$
P(\lambda) \rightarrow 3 x^{2} h_{1}
$$

In fact, for any value of $\lambda, P(\lambda)$ could be closely approached by a function proportional to $x^{2}$.

In the case of an oblate cavity, the situation is somewhat different. Indeed, in the case of a sandwich ( $e \rightarrow 1$ or equivalently $\lambda \rightarrow+\infty, P(\lambda)$ ) has a finite value:

$$
P(\lambda) \rightarrow 6 h_{3}+\frac{9 \pi^{2}}{16} h_{2}
$$

Therefore, for this geometry, $P(\lambda)$ cannot be approximated with a function proportional to $x^{2}$. The idea suggested by Gologanu et al. [15] was to introduce a new variable $y$ defined as: 


$$
y=\frac{x}{1+\rho x}
$$

where $\rho$ is a constant ${ }^{7}$. Note that $y$ is proportional to $x$ for low values of $e$, but has a finite value in the case of a sandwich $(e \rightarrow 1)$ i.e. $x \rightarrow+\infty: y \rightarrow \frac{1}{\rho}$. The connection between the spherical void case (for which $P(\lambda)$ is proportional to $x^{2}$ ) and the sandwich case can be realized by considering $P(\lambda)$ proportional to $y^{2}$. Thus, it appears reasonable to introduce the following approximation:

$\mathcal{A} 2 P(\lambda)$ is replaced by $p^{2} u^{2}, p$ being a constant while $u=x$ in the case of a prolate cavity and $u=y$ for an oblate cavity.

Let us now consider the term $W(\lambda)$ (see (80)). Following Gologanu [17], we introduce the approximation:

$\mathcal{A} 3 W(\lambda)$ is replaced by $w u^{2}$, where $w$ is a constant.

Using approximations $\mathcal{A} 2$ and $\mathcal{A} 3$, the expression (24) of $d_{\text {eq }}^{2}$ becomes:

$$
<d_{e q}^{2}>_{\mathcal{E}(\lambda)}=A_{e q}^{2}-\frac{w^{2} u^{2}}{p^{2}}(\boldsymbol{A}: \mathbb{H}: \boldsymbol{Q})^{2}+\left\{p B-\frac{w}{p} \boldsymbol{A}: \mathbb{H}: \boldsymbol{Q}\right\}^{2} u^{2}
$$

The last approximation considered is:

$\mathcal{A} 4$ The term $u^{2}$ which multiplies $\frac{w^{2}}{p^{2}}(\boldsymbol{A}: \mathbb{H}: \boldsymbol{Q})^{2}$ in the above equation (94) is replaced by the constant $u_{1} u_{2}$.

Thus, the expression of $<d_{e q}^{2}>_{\mathcal{E}(\lambda)}$ is:

$$
<d_{e q}^{2}>_{\mathcal{E}(\lambda)}=A_{e q}^{2}-\frac{w^{2} u_{1} u_{2}}{p^{2}}(\boldsymbol{A}: \mathbb{H}: \boldsymbol{Q})^{2}+\left\{p B-\frac{w}{p} \boldsymbol{A}: \mathbb{H}: \boldsymbol{Q}\right\}^{2} u^{2}
$$

Note that $\mathcal{A} 4$ is a good approximation, since it can be shown that for purely deviatoric macroscopic deformation, the exact value of $\Pi(\boldsymbol{D})$ is recovered.

\subsection{Calculation of coefficients $\eta, \zeta, \kappa($ or $p=3 / \kappa), \chi$ and $\rho$}

We present the determination of the expressions of the coefficients $\eta, \zeta, p$ and $\chi$ which are involved in the expression of the macroscopic criterion (52) through the definition (48) of $\widetilde{\Sigma}_{e q}$. Let first consider the coefficient $p$ which is

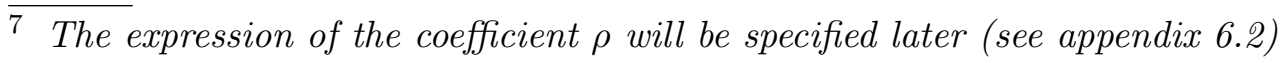


involved in the hyperbolic cosine term. In the case of an isotropic matrix, the method for determination of this coefficient proposed by Gologanu [14,15] was based on imposing certain conditions such as to obtain the exact solution in the particular case $\boldsymbol{A}=0$ which corresponds to a purely hydrostatic loading when the cavities are spherical. In fact, this is a loading path for which the plastic strain in the matrix is highly heterogeneous and the effect of $p$ is strong. The coefficient $p$ has been then calculated as follow:

$$
p=\frac{1}{\ln \left(u_{2} / u_{1}\right)} \int_{u_{1}}^{u_{2}} \sqrt{P(e)} \frac{d u}{u^{2}}
$$

Due to the fact that the integral in (96) cannot be estimated analytically, $\sqrt{P(e)}$ was approximated by a polynomial function.

In the anisotropic case, $P(e)$ is not only function of $e$ but also of anisotropy coefficients $h_{i}$ and the procedure proposed by Gologanu and collaborators proves to be difficult to implement. Since the term $P(e) / u^{2}$ is a function slightly oscillating with respect to $e$, we propose to replace it simply by its mean value on interval $\left[u_{1}, u_{2}\right] \cdot p$ is then given by:

$$
p^{2}=\frac{1}{u_{2}-u_{1}} \int_{u_{1}}^{u_{2}} P(\lambda) \frac{d u}{u^{2}}=-\frac{1}{u_{2}-u_{1}} \int_{\lambda_{1}}^{\lambda_{2}} P(\lambda) \frac{\left(2 a^{2}+b^{2}\right) b}{c^{3}} d \lambda
$$

The analytical computation of $p$ (or equivalently of $\kappa=3 / p$ ) by means of the second integral in (97) is very complicated and has been performed with the help of Maple Software. The obtained expression of $p$, valid either for prolate and oblate voids, takes the form:

$$
\begin{aligned}
& p^{2}=\frac{3}{2} \frac{(1+g)(f+g)}{f(1-f)}\left[\left(\beta_{1}-f \beta_{2}\right)\left(h_{1}+3 h_{2}-4 h_{3}\right)\right. \\
& \left.+6\left\{\alpha_{1}\left(1-\alpha_{1}\right)-f \alpha_{2}\left(1-\alpha_{2}\right)\right\} h_{2}+\left\{1-\alpha_{1}-f\left(1-\alpha_{2}\right)\right\}\left(h_{1}-3 h_{2}+4 h_{3}\right)\right]
\end{aligned}
$$

where the coefficients $h_{i}$, for $i=1,2,3$, are given by (84).

Concerning the coefficients $\eta$ and $\zeta$, their determination requires the evaluation of $w$. To estimate $w$, we use the procedure adopted for the determination of $p$. The mean value of $w$ on the interval $\left[u_{1}, u_{2}\right]$ is:

$$
w=\frac{2(1+g)(f+g)\left(\alpha_{2}-\alpha_{1}\right)}{\chi g(1-f)}
$$

$\eta$ and $\zeta$ are then derived based on (50). The result is given by (51). 
Coefficient $\rho$ which appears into the definition of $g$ is chosen such that $p$, given by (98), show the lowest variation according to $e_{1}$ and $e_{2}$. In [15] it has been proposed to consider the limited expression of $P(\lambda)$ for $\lambda \rightarrow+\infty$ (see (92)), nevertheless it has been observed that the consideration of function of eccentricity $e_{1}$ give better results and the following expression has been adopted:

$$
\rho=\frac{15 \pi}{8\left(1+\alpha_{1}^{2}\right)} \frac{h_{1}+3 h_{2}+4 h_{3}}{2 h_{1}+h_{2}+2 h_{3}}
$$

On figure 17 are represented $p$ with the previous expression for $\rho$ (100) (squares) and with the $\rho=4 / \sqrt{9 \pi^{2}+96}$ (circles) which correspond the limited expression of $P(\lambda)$ for $\lambda \rightarrow+\infty$ (see (92)). The low variation of $p$ according to $e_{1}$ by using (100) clearly justifies the choice made for this coefficient.

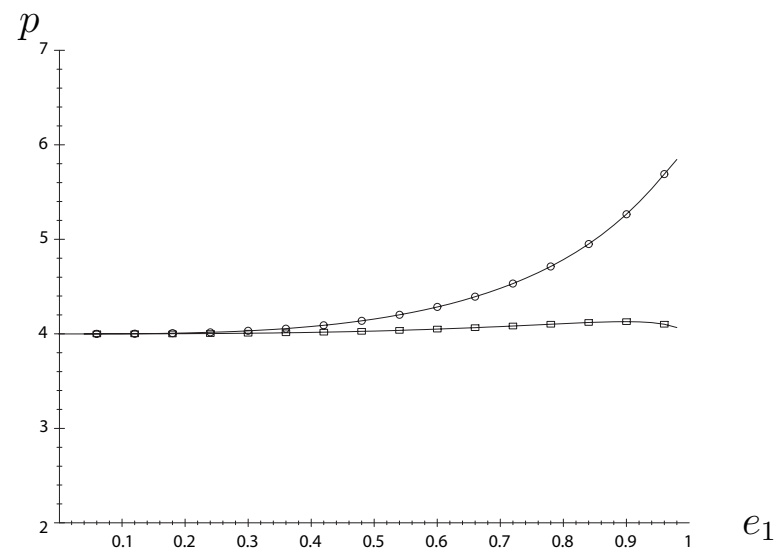

Fig. 17. Diagram giving coefficient $p$, as function of eccentricity $e_{1}$, for two values of coefficient $\rho$ and for the porosity $f=0.01$

\section{References}

[1] Benzerga, A.A., 2000. Rupture ductile des tôles anisotropes. PhD thesis, Ecole Nationale Supérieure des Mines de Paris.

[2] Benzerga, A.A., Besson, J., 2001. Plastic potentials for anisotropic porous solids. Eur. J. Mech. A/Solids 20, 397-434.

[3] Benzerga, A.A., Besson, J., Batisse, R., Pineau, A., 2002. Synergistic effects of plastic anisotropy and void coalescence on fracture mode in plane strain. Modelling Simul. Mater. Sci. Eng. 10, 73-102.

[4] Benzerga, A.A., Besson, J., Pineau, A., 2004. Anisotropic ductile fracture, Part I: experiments. Acta Materialia 152, 4623-4638.

[5] Benzerga, A.A., Besson, J., Pineau, A., 2004 Anisotropic ductile fracture, Part II: theory. Acta Materialia 152, 4639-4650. 
[6] Bonora, N., Gentile, D., Pirondi, A., Newaz, G., 2005. Ductile damage evolution under triaxial state of stress: theory and experiments. Int. J. Plasticity. 21, 981-1007.

[7] Brunet, M., Morestin, F., Walter, H., 2004. Damage identification for anisotropic sheet-metals using a non-local damage model. International Journal of Damage Mechanics, 13, 35-57.

[8] Budiansky, B., O'connell, R.J., 1976. Elastic moduli of a cracked solid. Int. J. Solid. Struct., 12(2) : 81-97.

[9] Chien, W. Y., Pan, J., Tang, S. C., 2001. Modified anisotropic Gurson yield criterion for porous ductile sheet metals. J. Eng. Mat. Tech. 123, 409-413.

[10] Croix, P., Lauro F., Oudin, J., Christlein, J., 2003. Improvement of damage prediction by anisotropy of microvoids. Journal of Materials Processing Technology 143-144, 202-208.

[11] Darrieulat, M., Montheillet, F., 1996. Extension of the Hill (1948) yield criterion to the case of prismatic monoclinic symmetry. Int. Journal of Mech. Science $38,1273-1284$.

[12] Gãrãjeu, M., 1995. Contribution à l'étude du comportement non linéaire de milieux poreux avec ou sans renfort. Ph.D. Thesis, University of Marseille, France.

[13] Garajeu, M., Michel, J.C., Suquet, P., 2000. A micromechanical approach of damage in viscoplastic materials by evolution in size, shape and distribution of voids, Comput. Methods Appl. Mech. Engrg. 183, 223-246.

[14] Gologanu, M., Leblond, J.-B., Devaux, J., 1993. Approximate models for ductile metals containing non-spherical voids - case of axisymmetric prolate ellipsoidal cavities. J. Mech. Phys. Solids 41 (11), 1723-1754.

[15] Gologanu, M., Leblond, J.-B., Devaux, J., 1994. Approximate models for ductile metals containing non-spherical voids - case of axisymmetric oblate ellipsoidal cavities. J. Eng. Mat. Tech. 116, 290-297.

[16] Gologanu, M., 1997. Etude de quelques problemes de rupture ductile, Ph.D. Thesis, University Pierre et Marie Curie, France.

[17] Gologanu, M., Leblond, J.-B., Perrin, G., Devaux, J., 1997. Recent extensions of Gurson's model for porous ductile metals, in: Suquet, P. (Ed.), Continuum Micromechanics, Springer, pp. 61-130.

[18] Gurson, A.L., 1977. Continuum theory of ductile rupture by void nucleation and growth: Part I. - Yield criterion and flow rules for porous ductile media. J. Eng. Mat. Tech. 99, 2-15.

[19] Hill, R., 1948. A theory of yielding and plastic flow of anisotropic solids. Proc. Roy. Soc. London A 193, 281-297. 
[20] Hom, C.L., McMeeking, R.M., 1989. Void growth in elastic-plastic materials. J. Appl. Mech. 56, pp. 309-317.

[21] Kysar, J.W. , Gan, Y.X., Mendez-Arzuza, G., 2005. Cylindrical void in a rigidideally plastic single crystal. Part I: Anisotropic slip line theory solution for face-centered cubic crystals. Int. J. Plasticity. 21(8), 1481-1520.

[22] Kysar, J.W. , Gan, Y.X., Morse, T.L., 2006. Cylindrical void in a rigid-ideally plastic single crystal II: Experiments and simulations. Int. J. Plasticity. 22(1), 39-72.

[23] Kysar, J.W. , Gan, Y.X., 2007. Cylindrical void in a rigid-ideally plastic single crystal III: Hexagonal close-packed crystal. Int. J. Plasticity. 23(4), 592-619.

[24] Leblond, J. B., Perrin, G., Suquet, P., 1994. Exact results and approximate models for porous viscoplastic solids. Int. J. Plasticity. 10(3), 213-235.

[25] Lee, B.I., Mear, M.E., 1992. Axisymmetric deformation of power-law solids containing a dilute concentration of aligned spheroidal voids. J. Mech. Phys. Solids 40, 1805-1837.

[26] Liao, K., Pan, J., Tang, S., 1997. Approximate yield criteria for anisotropic porous ductile sheet metals. Mech. Materials 26, 213-226.

[27] Liao, K., 2004. Yield criteria for porous ductile sheet metals with planar anisotropy under plane stress conditions. Computers and Structures 82, 25732583 .

[28] McClintock, F. A., 1968. A criterion for ductile fracture by the growth of holes. Approximate yield criteria for anisotropic porous ductile sheet metals. ASME J. Appl. Mech. 35, 363371.

[29] Monchiet, V., 2006. Contributions à la modélisation micromécanique de l'endommagement et de la fatigue des métaux ductiles. PhD thesis, University of Sciences and Technologies of Lille, France.

[30] Monchiet V., Charkaluk E., D. Kondo, 2007. An improvement of Gurson-type models of porous materials by using Eshelby-like trial velocity fields. C.R. Mécanique, 335, 32-41.

[31] Moon, P., Spencer, D.E., 1961. Field Theory Handbook. Springer-Verlag. Berlin.

[32] Pardoen, T., Hutchinson, J.W., 2000. An extended model for void growth and coalescence. J. Mech. Phys. Solids 48, 2467-2512.

[33] Pardoen, T., Hutchinson, J.W., 2003. Micromechanics-based model for trends in toughness of ductile metals. Acta Materialia 51, 133-148.

[34] Perrin, G., Leblond, J.-B., 1990. Analytical study of a hollow sphere made of plastic porous material and subjected to hydrostatic tension - application to some problems in ductile fracture of metals. Int. J. Plasticity, 6, 677-699.

[35] Perrin, G., 1992. Contribution à l'étude théorique et numérique de la rupture ductile des métaux. PhD Thesis, Ecole Polytechnique. 
[36] Pirondi, A., Bonora, N., Steglich, D., Brocks, W., Hellmann, D., 2006. Simulation of failure under cyclic plastic loading by damage models. Int. J. Plasticity. 22, 2146-2170.

[37] Ragab, A. R., Saleh, Ch. A. R., 1999. Evaluation of constitutive models for voided solids. International Journal of Plasticity, 15, 1041-1065.

[38] Ragab, A. R., 2004. Application of an extended void growth model with strain hardening and void shape evolution to ductile fracture under axisymmetric tension Engineering Fracture Mechanics 71, 1515-1534.

[39] Rice, J.R., Tracey D.M., 1969. On the ductile enlargement of voids in triaxial stress fields. J. Mech. Phys. Solids 17, 201-217.

[40] Siruguet K, Leblond, J-B., 2004. Effect of void locking by inclusions upon the plastic behavior of porous ductile solids - I: theoretical modeling and numerical study of void growth. Int. J. Plasticity. 20(2), 225-254.

[41] Siruguet K, Leblond, J-B., 2004. Effect of void locking by inclusions upon the plastic behavior of porous ductile solids - II: theoretical modeling and numerical study of void growth. Int. J. Plasticity. 20(2), 255-268.

[42] Son, H.S., Kim, Y.S., 2003. Prediction of forming limits for anisotropic sheets containing prolate ellipsoidal voids. Int. J. Mech. Sci. 45, 1625-1643.

[43] Suquet, P., 1982. Plasticité et homogénéisation. PhD. Thesis, University Pierre et Marie Curie.

[44] Tvergaard, V., Needleman, A., 1984. Analysis of the cup-cone fracture in a round tensile bar. Acta Met. Mater. 32, 157-169.

[45] Wang, D., Pan, J., 2004. An Anisotropic Gurson Yield Criterion for Porous Ductile Sheet Metals with Planar Anisotropy. International Journal of Damage Mechanics, Vol. 13. 